\title{
RECENTES AVANÇOS NA FUNCIONALIZAÇÃO SELETIVA DE QUINOLINAS
}

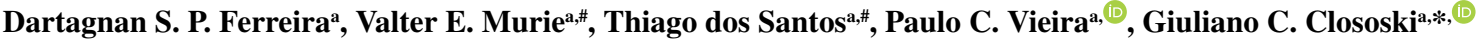 \\ aDepartamento de Ciências Biomoleculares, Faculdade de Ciências Farmacêuticas de Ribeirão Preto, Universidade de São Paulo, \\ 14040-903 Ribeirão Preto - SP, Brasil
}

Recebido em 23/10/2020; aceito em 04/12/2020; publicado na web em 20/01/2021

\begin{abstract}
RECENT ADVANCES IN SELECTIVE FUNCTIONALIZATION OF QUINOLINES. Heterocyclic compounds form an important and extensive group of organic substances. Among nitrogenous heterocyclic molecules, quinolines stand out for exhibiting attractive chemical and biological properties. These substances can be used as ligands, sensors, luminescent and agrochemical materials. In addition, quinoline-containing compounds can exhibit a wide spectrum of pharmacological properties, allowing their use in several approved drugs nowadays. Due to its importance, the synthesis of molecules containing this nucleus becomes a point of interest for synthetic chemists. In this way, several methodologies have been recently developed to prepare quinoline derivatives with high structural diversity. Such chemical transformations allow the chemical modification of these rings with high selectivity and tolerance to diverse functional groups and these properties have been conveniently used in the preparation of biologically active molecules containing this unit. Herein, we present a review of the main methodologies employed in the selective functionalization of quinolines in the last twenty years. In this context, a brief introduction addressing general synthetic and medicinal aspects related to the functionalization positions of the quinoline ring is presented. Several methodologies used in the functionalization of this moiety are discussed, as well relevant synthetic applications, both in the preparation and functionalization of substances of biological interest.
\end{abstract}

Keywords: quinoline; $N$-heterocycles; selective-metalation; C-H functionalization.

\section{INTRODUÇÃo}

Os compostos heterocíclicos formam um importante e extenso grupo de substâncias orgânicas. Além de desempenharem um papel fundamental em diversos sistemas bioquímicos, eles possuem grande aplicabilidade nas áreas farmacêutica, agroquímica e industrial. ${ }^{1-3} \mathrm{Em}$ particular, de acordo com o banco de dados da U.S. FDA (United States Food and Drug Administration), os compostos heterocíclicos nitrogenados estão presentes em mais da metade dos medicamentos aprovados. ${ }^{4}$ Dentre as substâncias heterocíclicas nitrogenadas, as quinolinas, compostos que se caracterizam pela presença de um anel benzênico e um piridínico fundidos, também chamadas de 1-azanaftaleno, 1-benzazina, ou benzo $[b]$ piridina, se destacam por exibirem atraentes propriedades químicas e biológicas.

De forma geral, compostos contendo essa unidade bicíclica podem ser encontrados na forma de produtos naturais ou mesmo na estrutura molecular de fármacos. ${ }^{5}$ Além disso, quinolinas podem ser empregadas como ligantes em complexos metálicos, ${ }^{6,7}$ agente geleificante, ${ }^{8}$ sondas fluorescentes,,${ }^{9,10}$ em células solares sensibilizadas por corante ${ }^{11} \mathrm{e}$ na fabricação de polímeros com potencial aplicação em materiais eletrônicos, termorresistentes e microfibrosos dopados com metais na superfície. ${ }^{12,13}$ É importante destacar que moléculas contendo essa unidade podem apresentar amplo espectro de atividades biológicas como atividade antiploriferativa, ${ }^{14-20}$ antibacteriana, ${ }^{21,22}$ leishmanicida, ${ }^{23}$ antiviral, ${ }^{24-26}$ antichagásica, ${ }^{27}$ antimalárica, ${ }^{28,29}$ inibitória de catepsina $\mathrm{K}^{30}$ e inseticida. ${ }^{31,32}$ A Figura 1 apresenta de forma geral alguns aspectos medicinais relacionados às posições de funcionalização do anel quinolínico.

As interessantes propriedades medicinais exibidas pelos derivados quinolínicos permitiram o desenvolvimento de vários fármacos, dentre os quais podem ser destacados o cabozantinibe, ${ }^{33}$ lenvatinibe ${ }^{34}$ cloroquina, ${ }^{35}$ mefloquina, ${ }^{36}$ tafenoquina $^{37}$ e montelucaste $^{38}$ (Figura 2).

*e-mail: gclososki@usp.br

"Estes autores contribuíram igualmente com o artigo.
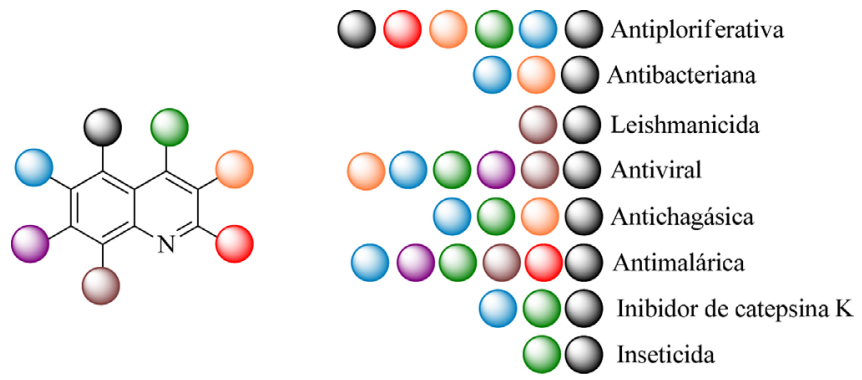

Figura 1. Atividades relacionados às posições de funcionalização do anel quinolínico

Dada a importância do grupo quinolina, a síntese de moléculas contendo este núcleo passa a ser um ponto de interesse por parte dos químicos sintéticos. É nesse contexto que diversas metodologias têm sido recentemente desenvolvidas com o objetivo de preparar derivados quinolínicos com diferentes funcionalizações. Em particular, a funcionalização seletiva de anéis quinolínicos tem apresentado significantes avanços nas últimas duas décadas. Tais transformações químicas permitem a funcionalização desses anéis com alta seletividade e tolerância a grupos funcionais diversos e estas propriedades têm sido convenientemente utilizadas na preparação de moléculas biologicamente ativas contendo esse núcleo.

Desta forma, este trabalho tem como objetivo apresentar uma revisão crítica das principais metodologias empregadas na funcionalização seletiva de quinolinas, abordando relevantes aplicações sintéticas dessas transformações químicas, tanto na preparação como na funcionalização seletiva de substâncias de interesse biológico contendo esta unidade. Para isso, foi realizado um levantamento bibliográfico das metodologias desenvolvidas nas últimas duas décadas (2000-2020). É importante destacar que em relação às reações de funcionalização $\mathrm{C}-\mathrm{H}$ de anéis quinolínicos o levantamento feito nesta revisão compreendeu o período correspondente aos últimos cinco anos, tendo em vista os recentes 
<smiles>CCN(CC)CCCC(C)Nc1ccnc2cc(Cl)ccc12</smiles>

Figura 2. Estruturas de alguns fármacos contendo o núcleo quinolínico

artigos de revisão publicados que abordam tal transformação química. $^{39-42}$

\section{FUNCIONALIZAÇÃO SELETIVA DE QUINOLINAS}

\section{Metalação dirigida e troca halogênio-metal}

Compostos heteroaromáticos funcionalizados podem, sob determinadas condições, ser convenientemente preparados pela reação de metalação dirigida de heteroarenos. ${ }^{43-47}$ Em particular, esse método sintético permite a desprotonação de compostos quinolínicos através da utilização de bases fortes não nucleofílicas, onde os amidetos de lítio e reagentes do tipo alquil-lítio são tradicionalmente empregados com esse objetivo. ${ }^{48}$ De maneira geral, as limitações da metodologia residem na alta reatividade de alguns reagentes do tipo alquil-lítio frente aos anéis quinolínicos bem como à baixa estabilidade dos reagentes organolítio em solução. ${ }^{49}$ Assim, o manuseio desses reagentes frequentemente requer o uso de baixas temperaturas reacionais, sendo este um fator limitante no escalonamento das reações de metalação dirigida de quinolinas bem como em aplicações sintéticas adicionais. ${ }^{50-52}$ Apesar das limitações citadas anteriormente, as bases orgânicas de lítio podem promover reações de metalação de anéis quinolínicos com alta regiosseletividade, sob condições reacionais específicas. Esse fato foi observado por Schlosser e Marull durante estudos referentes à metalação de quinolinas promovida por diferentes bases de lítio. ${ }^{53}$ Neste trabalho, quinolinas $(\mathbf{1}, \mathbf{4}$ e $\mathbf{7})$ contendo o substituinte $-\mathrm{CF}_{3}$ foram submetidas à reação de metalação utilizando bases LDA, LiTMP (2,2,6,6-tetrametilpiperidin-1-eto de lítio) e $n$-BuLi, seguido da adição de gás $\mathrm{CO}_{2}$ como eletrófilo e posterior etapa de acidificação. Dessa forma, produtos contendo o grupo carboxílico em diversas posições do anel quinolínico foram obtidos regiosseletivamente e em rendimentos variados (Esquema 1).

Nos últimos anos, amidetos de magnésio têm sido amplamente empregados na funcionalização de diversos arenos e heteroarenos. ${ }^{54-63}$ Bases desse tipo possuem alta reatividade e são solúveis em THF quando na presença de $\mathrm{LiCl}$, formando amidetos mistos de magnésio e lítio. Além disso, esses reagentes apresentam maior estabilidade em meio reacional quando comparados com amidetos de lítio, permitindo que reações de metalação dirigida de quinolinas sejam realizadas em condições reacionais mais brandas, na presença de grupos<smiles>CC(C)(C)c1ccc2cccc(C(=O)O)c2n1</smiles><smiles></smiles><smiles>O=C(O)c1cc(C(F)(F)F)c2ccccc2n1</smiles><smiles>FC(F)(F)c1ccc2ccccc2n1</smiles><smiles>FC(F)(F)c1cnc2ccccc2c1</smiles>

4<smiles>FC(F)(F)c1ccnc2ccccc12</smiles>

7

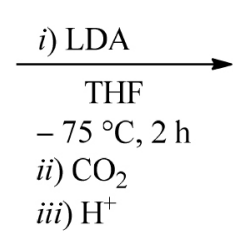<smiles>O=C(O)c1cc2ccccc2nc1C(F)(F)F</smiles>

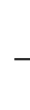

i) LDA

$-75^{\circ} \mathrm{C}, 2 \mathrm{~h}$

ii) $\mathrm{CO}_{2}$

iii) $\mathrm{H}^{+}$<smiles>O=C(O)c1c(C(F)(F)F)cnc2ccccc12</smiles><smiles>O=C(O)c1cnc2ccccc2c1C(F)(F)F</smiles> 
funcionais diversos. ${ }^{64}$ Nesse contexto, Knochel e colaboradores estudaram o controle da regiosseletividade das reações de metalação do anel quinolínico presente na estrutura molecular da quinina (10), um alcaloide natural com atividade antimalárica. ${ }^{65}$ Nesse estudo, verificou-se que após uma etapa de proteção do grupo hidroxila presente em 10, a metalação na presença deste alcaloide e um ácido de Lewis levou à formação dos derivados sintéticos da quinina 2-funcionalizados 11a-b em rendimentos moderados, após adição do eletrófilo correspondente. ${ }^{66}$ A elevada regiosseletividade observada nesta reação é influenciada pela presença do volumoso éter de silício, gerado por meio da reação do álcool secundário com TBDMSCl (Esquema 2).

Por outro lado, a funcionalização da quinina na posição C3 do anel quinolínico correspondente foi alcançada quando $\mathbf{1 0}$ foi submetido inicialmente a uma etapa de desprotonação do grupo hidroxila usando $\mathrm{MeLi}$, na presença de $\mathrm{BF}_{3} \cdot \mathrm{OEt}_{2}$. Após essa etapa, reações de metalação seguidas pelas adições dos eletrófilos apropriados levaram aos derivados de quinina 12a-b, em bons rendimentos. Além do controle de regiosseletividade, ambas metodologias empregaram condições reacionais brandas.

A utilização de grupos dirigentes de metalação (GDM) tem se destacado por permitir a metalação dirigida de arenos e heteroarenos com alta regiosseletividade. Substituintes como halogênio, calcogênio, carboxilato e amida são alguns dos diversos grupos dirigentes de metalação empregados atualmente para essa finalidade. ${ }^{43,48,67,68} \mathrm{Em}$ particular, o grupo fosforodiamidato tem sido utilizado como GDM de forma eficiente em reações de metalação de quinolinas. Esse grupo orto-dirigente de metalação permite a complexação deste núcleo com os amidetos mistos de $\mathrm{Mg} / \mathrm{Li} .^{69}$ Além de dirigir este tipo de reação, o grupo dirigente fosforado pode ser posteriormente removido através de uma hidrólise. Em 2010, Knochel e colaboradores empregaram o grupo fosforodiamidato como GDM nas reações de metalação sucessivas visando à preparação de moléculas de interesse biológico. ${ }^{70}$ Nesse trabalho, os intermediários sintéticos $\mathbf{1 4 a - b}$ foram inicialmente preparados em bons rendimentos a partir da reação de magnesiação de 13 promovida por $\mathrm{TMP}_{2} \mathrm{Mg} \cdot 2 \mathrm{LiCl}$, seguida de transmetalação e acoplamento de Negishi catalisado por paládio. Uma segunda reação de metalação foi utilizada na obtenção das quinolinas 15a-b a partir de 14a-b. Nessa etapa de síntese, TMPMgCl-LiCl foi empregado como base e as quinolinas $\mathbf{1 5 a - b}$ foram obtidas em bons rendimentos, através da reação de magnesiação à temperatura ambiente com adição de $\mathrm{NC}-\mathrm{CO}_{2}$ Et como eletrófilo (Esquema 3).

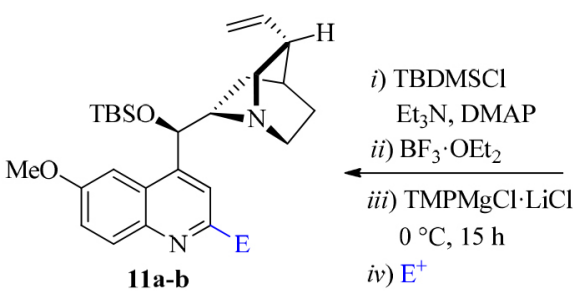

alguns compostos sintetizados<smiles>C=C[C@H]1CN2CC[C@@H]1[C@H]2[C@H](O)c1ccnc2ccc(OC)cc12</smiles>

i) $\mathrm{MeLi}$

ii) $\mathrm{BF}_{3} \cdot \mathrm{OEt}_{2}$

iv) $\mathrm{E}^{+}$

12a-b

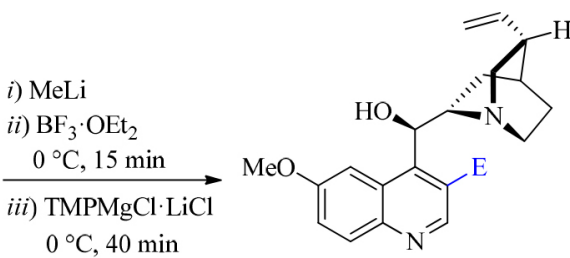

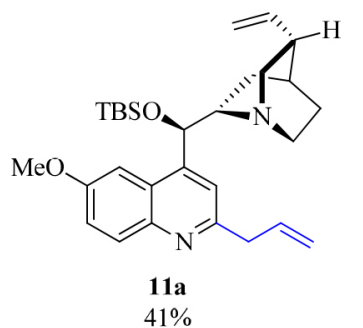<smiles>C=C[C@H]1CN2CC[C@@H]1[C@H]2[C@H](O[GaH])c1cc(I)nc2ccc(OC)cc12</smiles>

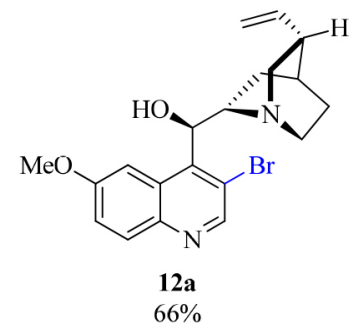

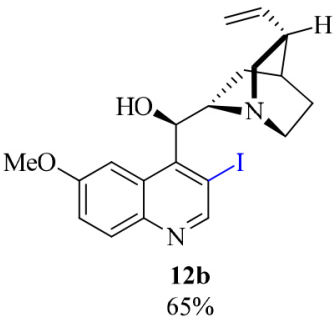

Esquema 2. Funcionalização regiosseletiva da quinina (10)<smiles>CNP(=O)(Oc1cnc2ccccc2c1)N(C)C</smiles>

13

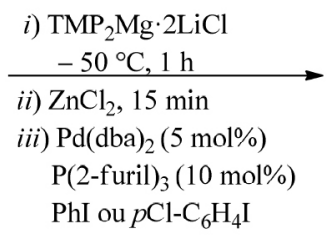

i) $\mathrm{TMP}_{2} \mathrm{Mg} \cdot 2 \mathrm{LiCl}$
$-50^{\circ} \mathrm{C}, 1 \mathrm{~h}$

ii) $\mathrm{ZnCl}_{2}, 15 \mathrm{~min}$ $\mathrm{P}(2 \text {-furil })_{3}(10 \mathrm{~mol} \%)$ $\mathrm{PhI}$ ou $p \mathrm{Cl}-\mathrm{C}_{6} \mathrm{H}_{4} \mathrm{I}$<smiles>[R]c1ccc(-c2nc3ccccc3cc2OP(N)(=O)N(C)C)cc1</smiles>

14a.: $\mathrm{R}=\mathrm{H}(81 \%)$ 14b.: $\mathrm{R}=\mathrm{Cl}(78 \%)$ i) $\mathrm{TMPMgCl} \cdot \mathrm{LiCl}$<smiles>[R]c1ccc(-c2nc3ccccc3cc2O)cc1</smiles>

15a.: $\mathrm{R}=\mathrm{H}(79 \%)$ 15b.: $\mathrm{R}=\mathrm{Cl}(81 \%)$<smiles>CCC(NC(=O)c1c(O)c(-c2ccccc2)nc2ccccc12)C(=O)N[C@@H](CC)c1ccccc1</smiles>

Esquema 3. Metalações sucessivas na preparação de moléculas de interesse biológico 
A partir do intermediário sintético $\mathbf{1 5 a}$, foi possível a preparação do talnetant (16), um antagonista do receptor NK3. ${ }^{71}$ Adicionalmente, a quinolina 15b foi empregada na obtenção de um inibidor de P-selectina (17), preparado em bom rendimento através de duas etapas sintéticas. Nessa síntese, a base $\mathrm{TMP}_{2} \mathrm{Mg} \cdot 2 \mathrm{LiCl}$, na presença de $\mathrm{ZnCl}_{2}$, foi empregada na zincação da posição $\mathrm{C} 8$ de $\mathbf{1 5 b}$, permitindo a arilação dessa posição após acoplamento de Negishi. Sendo assim, foi possível a obtenção de $\mathbf{1 7}$ após uma etapa adicional de remoção do grupo GDM.

Amidetos de zinco também podem ser empregados na reação de metalação dirigida de quinolinas. Sua utilização nesse tipo de transformação química tem permitido a funcionalização seletiva de quinolinas através de reações como, por exemplo, acoplamento cruzado de Negishi, homologação, metalação e hidroxilação. ${ }^{72}$ Tendo em vista as diferentes reatividades dos amidetos de lítio, magnésio e zinco, Clososki e colaboradores demonstraram interesse no estudo da regiosseletividade das reações de metalação dirigida da 4,7-dicloroquinolina 18 (4,7-DCQ), empregando diferentes amidetos metálicos. Nesses estudos, derivados 4,7-DCQ 3-funcionalizados (19a-b) foram preparados pelo uso de LDA como base e um eletrófilo, à temperatura de $-70{ }^{\circ} \mathrm{C}$ (Esquema 4$){ }^{73}$

A alta regiosseletividade desta transformação química se deve à abstração preferencial do hidrogênio mais ácido do substrato. Por outro lado, metalação à temperatura ambiente empregando $\mathrm{TMPMgCl} \cdot \mathrm{LiCl}$ e TMPZnCl$\cdot \mathrm{LiCl}$ forneceu derivados 4,7-DCQ 8-funcionalizados em rendimentos variando de moderados a bons, após adição dos eletrófilos correspondentes. Nesse caso, a funcionalização $\mathrm{C} 8$ foi possível devido à coordenação do nitrogênio quinolínico com os metais $\mathrm{Mg}$ e $\mathrm{Zn}$, o que causa a diminuição do valor de $\mathrm{pK}_{\mathrm{a}}$ do hidrogênio desta posição, como indicado por cálculos de DFT (do inglês, Density Functional Theory). Vale destacar que a funcionalização nessa posição $(\mathrm{C} 8)$ é importante na obtenção de quinolinas com propriedades antiviral, antimalárica e leishmanicida (Figura 1).

Reações de troca halogênio-metal constituem um método alternativo na funcionalização de compostos quinolínicos. Reagentes organometálicos do tipo $\mathrm{Bu}_{3} \mathrm{MgLiBr}, n$-BuLi e $\mathrm{MesMgBr} \cdot \mathrm{LiCl}$ podem ser empregados para essa finalidade. ${ }^{74-77} \mathrm{Em}$ particular, o reagente turbo-Grignard (iPrMgCl$\cdot \mathrm{LiCl})$ tem sido amplamente empregado em reações de troca halogênio-magnésio de diversos substratos halogenados. ${ }^{78-81} \mathrm{~A}$ importância desta reação na funcionalização de quinolinas pode ser ilustrada pelo trabalho de Baron e Knochel, que realizaram a síntese do derivado quinolínico 23 através de duas etapas de troca I/Mg. Inicialmente, o reagente de partida $\mathbf{2 1}$ foi submetido a uma reação de troca halogênio-metal promovida por iPrMgCl, em THF como solvente (Esquema 5). ${ }^{82}$

Essa reação ocorreu de forma regiosseletiva na posição C7 do anel quinolínico de 21 e, assim, após adição do eletrófilo pinacol borato, foi possível a preparação do intermediário sintético 22 em $81 \%$ de rendimento. Em seguida, o reagente turbo-Grignard foi utilizado na segunda reação de troca $\mathrm{I} / \mathrm{Mg}$, seguido de posterior adição de $\mathrm{CuCN} \cdot 2 \mathrm{LiCl}$. Após formação do organocuprato correspondente, 3-bromopropeno foi adicionado como eletrófilo, fornecendo o produto de reação $\mathbf{2 3}$ em excelente rendimento reacional.

Chen e colaboradores empregaram a reação de troca $\mathrm{Br} / \mathrm{Li}$ na preparação de um dos diastereoisômeros da mefloquina $\left(\right.$ Lariam $\left.^{\circledR}\right)$, um medicamento utilizado no tratamento da malária. ${ }^{83}$ Os autores realizaram inicialmente uma reação de troca $\mathrm{Br} / \mathrm{Li}$ a partir da quinolina 24, empregando $n$-BuLi como base em $\mathrm{Et}_{2} \mathrm{O}$ como solvente sob baixas temperaturas. Após adição do aldeído 25, foi possível a preparação do intermediário sintético $\mathbf{2 6}$ com razão diastereoisomérica superior a 95:5. Após etapa de remoção do grupo protetor Boc e acidificação, o cloridrato de (-)-eritro-mefloquina (27) foi obtido em $88 \%$ de rendimento (Esquema 6).

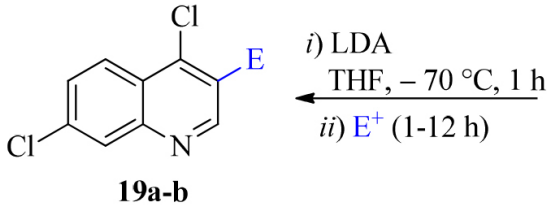

alguns compostos sintetizados<smiles>O=Cc1cnc2cc(Cl)ccc2c1Cl</smiles><smiles>OC(c1ccccc1Br)c1cnc2cc(Cl)ccc2c1Cl</smiles><smiles>Clc1ccc2c(Cl)ccnc2c1</smiles>

18<smiles>Clc1ccc2c(Cl)ccnc2c1I</smiles>

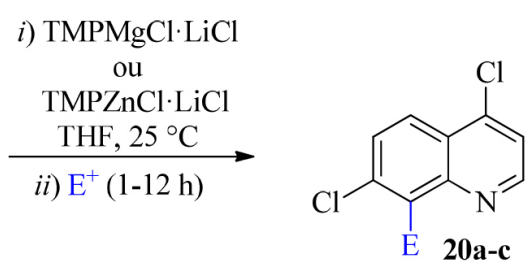<smiles>OC(c1ccc(F)cc1)c1c(Cl)ccc2c(Cl)ccnc12</smiles><smiles>O=Cc1c(Cl)ccc2c(Cl)ccnc12</smiles>

Esquema 4. Controle da regiosseletividade de reações de metalação dirigida de 4,7-DCQ (18)<smiles>FC(F)(F)c1c(I)cc(I)c2cccnc12</smiles>

$$
\begin{aligned}
& \text { i) } \mathrm{iPrMgCl}, \mathrm{THF} \text {, } \\
& \text { ii) } \mathrm{B} \text { (pin)OEt } \\
& -78^{\circ} \mathrm{C} \rightarrow \mathrm{ta}
\end{aligned}
$$

21<smiles>CC1(C)OB(c2cc(I)c3cccnc3c2O[Na])OC1(C)C</smiles>

$81 \%$ i) $\mathrm{iPrMgCl} \cdot \mathrm{LiCl}$

$\stackrel{\text { ii) } \mathrm{CuCN} \cdot 2 \mathrm{LiCl}}{\longrightarrow}$

iii)

THF

$\mathrm{Br}$<smiles>C=CC[Bi]</smiles><smiles>C=CCc1cc(B2OC(C)(C)C(C)(C)O2)c(O[Na])c2ncccc12</smiles>

$91 \%$

Esquema 5. Reações de trocas $\mathrm{I} / \mathrm{Mg}$ de quinolinas promovidas por iPrMgCl e iPrMgClLiCl 
<smiles>FC(F)(F)c1cc(Br)c2cccc(C(F)(F)F)c2n1</smiles>

i) $n$ - $\mathrm{BuLi}$

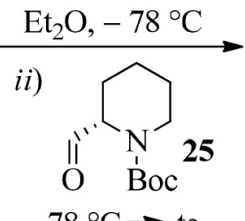

24

$$
-78^{\circ} \mathrm{C} \rightarrow \text { ta }
$$

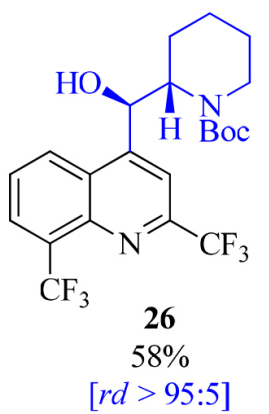<smiles>OC(c1cc(C(F)(F)F)nc2c(C(F)(F)F)cccc12)C1CCCCN1Cl</smiles>

[(-)-eritro-mefloquina]

Esquema 6. Síntese do cloridrato (-)-eritro-mefloquina (27) via troca $\mathrm{Br} / \mathrm{Li}$

A régio- e quimiosseletividade das reações de troca $\mathrm{Br} / \mathrm{Mg}$, em conjunto com as metalações dirigidas de quinolinas promovida por amidetos mistos de magnésio e lítio, foram exploradas por Knochel e colaboradores na preparação do talnetant (16).${ }^{77}$ Neste trabalho, a dibromoquinolina $\mathbf{2 8}$ foi inicialmente submetida à reação de troca $\mathrm{Br} / \mathrm{Mg}$ na presença de $\mathrm{iPrMgCl} \cdot \mathrm{LiCl}$. Essa etapa de magnesiação ocorreu com alta regiosseletividade e após adição do eletrófilo correspondente foi possível a obtenção da bromoquinolina 29 , em rendimento excelente. Em seguida, foi realizada uma etapa de metalação dirigida de 29 empregando $\mathrm{TMPMgCl} \cdot \mathrm{LiCl}$ como base. Essa reação foi conduzida em condições reacionais brandas, e após adição do pinacol borato como eletrófilo e posterior acidificação, obteve-se o intermediário sintético 30 em $71 \%$ de rendimento. A partir desse intermediário (30), talnetant (16) foi preparado em rendimento moderado após três etapas sintéticas adicionais (Esquema 7).

\section{Funcionalização C-H}

Nos últimos anos, as reações de ativação C-H têm sido empregadas de forma alternativa na funcionalização seletiva de quinolinas. Essas reações permitem a modificação de ligações químicas do tipo $\mathrm{C}-\mathrm{H}$ relativamente inertes, com alta seletividade e tolerância a diversos grupos funcionais. ${ }^{40,41}$ Nesse contexto, as reações de borilação catalisadas por irídio têm se apresentado como um dos principais métodos de ativação C-H de heterociclos. ${ }^{84-87} \mathrm{Em}$ 2012, uma metodologia de ativação C-H de compostos aromáticos e quinolínicos foi desenvolvida por Steel e colaboradores utilizando pequenas quantidades de $[\operatorname{Ir}(\mathrm{OMe}) \operatorname{cod}]_{2}(33)$ como catalisador e<smiles>Brc1cc(Br)c2ccccc2n1</smiles>

28

$$
\begin{aligned}
& \text { i) } \mathrm{iPrMgCl} \cdot \mathrm{LiCl} \\
& \stackrel{\mathrm{THF},-78{ }^{\circ} \mathrm{C}, 2 \mathrm{~h}}{\longrightarrow} \\
& \underset{\text { ii) } \mathrm{NC}-\mathrm{CO}_{2} \mathrm{Et}}{-78}{ }^{\circ} \mathrm{C} \rightarrow \mathrm{ta}, 12 \mathrm{~h}
\end{aligned}
$$<smiles>CCOC(=O)c1c(B2OC(C)(C)C(C)(C)O2)c(Br)[n+]([Al])c2ccccc12</smiles>

dtbpy (34), na presença de $\mathrm{B}_{2}$ (pin) $)_{2}$ em MTBE como solvente. ${ }^{88}$ Os autores investigaram os efeitos estéricos e eletrônicos envolvidos nessas reações, chegando inclusive a realizar previsões relacionadas à regiosseletividade destas através do deslocamento químico de hidrogênios ligados diretamente a anéis quinolínicos com diferentes padrões de substituição, por espectroscopia de RMN de ${ }^{1} \mathrm{H}$. De forma geral, essa metodologia se mostrou eficiente na funcionalização de quinolinas dissubstituídas, fornecendo produtos de ativação $\mathrm{C}-\mathrm{H}$ em bons rendimentos. Além disso, essa metodologia apresentou alta tolerância a diferentes grupos funcionais, alta regiosseletividade e excelente conversão química. Vale destacar que o respectivo protocolo permitiu a instalação do grupo boronato no anel quinolínico, uma funcionalidade bastante útil quando modificações químicas posteriores são desejadas (Esquema 8).

Recentemente, Wang e colaboradores desenvolveram uma metodologia sintética de alquilação de ligação C-H em compostos quinolínicos, empregando um fotocatalisador de irídio. ${ }^{89}$ Nesse trabalho, a funcionalização de quinolinas 4-substituídas foi realizada empregando apenas $1 \mathrm{~mol} \% \mathrm{de} \operatorname{Ir}\left[\mathrm{dF}\left(\mathrm{CF}_{3}\right) \mathrm{ppy}\right]_{2}(\mathrm{dtbbpy}) \mathrm{PF}_{6},(\mathbf{3 8}) \mathrm{um}$ catalisador contendo irídio III coordenado com 3 ligantes bipiridínicos com diferentes substituições. Além disso, foram empregados nesta reação $t$-BPA, TFA e um iodeto de alquila. Essa metodologia permitiu a síntese dos híbridos quinolínicos 39a-b em bons rendimentos, empregando condições reacionais brandas (Esquema 9 - condição 1).

No mesmo período, este grupo de pesquisa desenvolveu uma metodologia de alquilação C-H de quinolinas empregando oxalatos (Esquema 9 - condição 2)..$^{90}$ Nesse segundo trabalho, Wang e colaboradores realizaram funcionalizações remotas de quinolinas

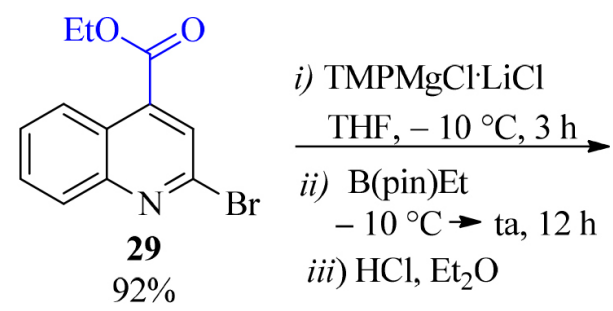<smiles>CCC(NC(=O)c1c(O)c(-c2ccccc2)nc2ccccc12)c1ccccc1</smiles>

[talnetant] 
<smiles>[R]c1ccc2nc([R11]([H])([H])[H])ccc2c1</smiles>

Esquema 8. Borilação de quinolinas 4,7- e 2,6- dissubstituídas

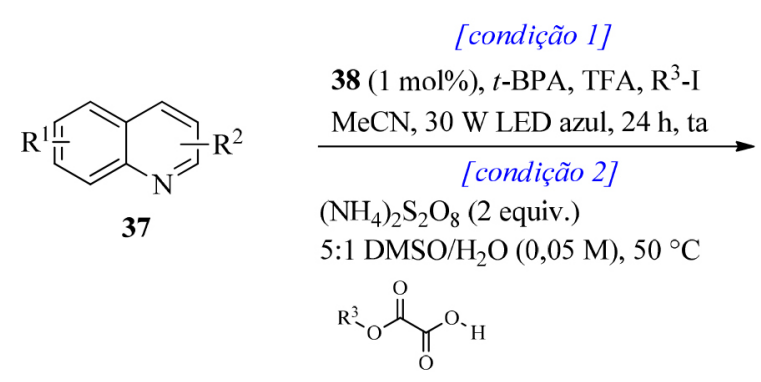

alguns compostos sintetizados

[condição 1]
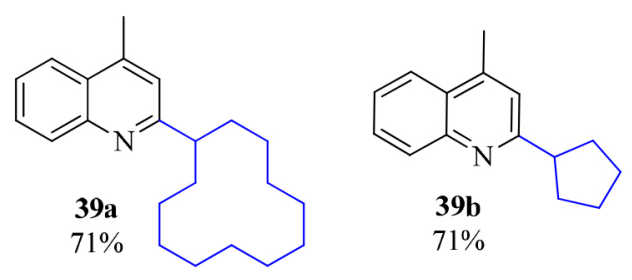

Esquema 9. Alquilação $\mathrm{C}$-H de derivados quinolínicos

presentes na estrutura molecular de produtos naturais e farmacêuticos na ausência de metais, fotocatalisadores ou radiação, empregando persulfato como agente oxidante, na presença de oxalatos com diferentes substituintes. Este protocolo não exigiu altas temperaturas nem excesso de substrato, sendo uma importante alternativa na funcionalização de quinolinas de interesse biológico. Ambas as metodologias mencionadas anteriormente permitiram a alquilação C-H na posição C2 de anéis quinolínicos substituídos através de radicais alquílicos gerados in situ, a partir de iodetos ou álcoois correspondentes. Funcionalizações nessa posição do anel quinolínico estão relacionadas com as mais variadas atividades biológicas relacionadas a esta unidade bicíclica (Figura 1).

Em 2016, Kanai e colaboradores desenvolveram uma metodologia de funcionalização $\mathrm{C}-\mathrm{H}$ através da trifluorometilação de diversos derivados quinolínicos, uma importante transformação explorada em química medicinal. ${ }^{91}$ Nesse trabalho, várias quinolinas reagiram na presença de $\mathrm{B}\left(\mathrm{C}_{6} \mathrm{~F}_{4}-4-\mathrm{CF}_{3}\right)_{3}$, um ácido de Lewis gerado in situ a partir da reação de troca $\mathrm{Br} / \mathrm{Li}$ de $\mathbf{4 0}$ seguido da adição de $\mathrm{BCl}_{3}$ como eletrófilo. Com isso, foi possível a obtenção dos adutos quinolínicos 42a-e em rendimentos variados. A formação desses adutos permitiu a funcionalização na posição $\mathrm{C} 4$ do anel quinolínico devido a

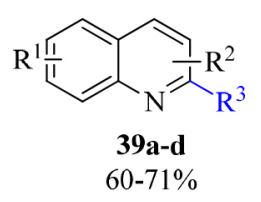

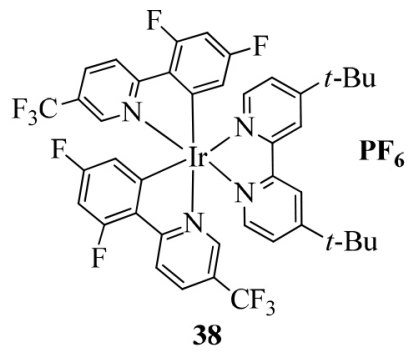<smiles>C=C[C@]1(C)CN2CC[C@H]1C2[C@H](O)c1cc(C(C)(C)C)nc2ccc(OC)cc12</smiles>

ativação causada pela coordenação entre o átomo de nitrogênio desse heterociclo com $\mathrm{B}\left(\mathrm{C}_{6} \mathrm{~F}_{4}-4-\mathrm{CF}_{3}\right)_{3}$. Desta forma, 42a-e foram submetidos à reação de trifluorometilação com $\mathrm{Me}_{3} \mathrm{SiCF}_{3}$ e TBAT, em acetato de etila como solvente, levando à formação de intermediários sintéticos contendo o grupo $-\mathrm{CF}_{3}$ na posição $\mathrm{C} 4$. Após essa etapa, foi realizada uma reação de oxidação na presença de $\mathrm{PhI}\left(\mathrm{O}_{2} \mathrm{CCF}_{3}\right)_{2}$, fornecendo as quinolinas 43a-e com rendimentos variando de moderado a bons (Esquema 10).

Poucos anos atrás, Baidya e colaboradores desenvolveram uma metodologia sintética de funcionalização $\mathrm{C}-\mathrm{H}$ de diversas quinolinas empregando catalisadores de cobre..$^{92}$ Nesse sentido, 8-amidoquinolinas foram submetidas à reação com a uma $\mathrm{N}$-halossuccinimida (NIS ou NBS), na presença de quantidades catalíticas de $\mathrm{Cu}(\mathrm{OTf})_{2}$, em DME como solvente. Essa etapa forneceu 5-haloquinolinas 46a-d com alta regiosseletividade e bons rendimentos, permitindo inclusive a obtenção de um desses produtos (46a) em pouco mais de 1 grama em massa. De forma geral, essa metodologia permitiu a reação de bromação e iodação de várias quinolinas com alta tolerância a diversos grupos funcionais, através de um protocolo simples e robusto. Como aplicação sintética, os autores empregaram a metodologia desenvolvida nesse trabalho na preparação 


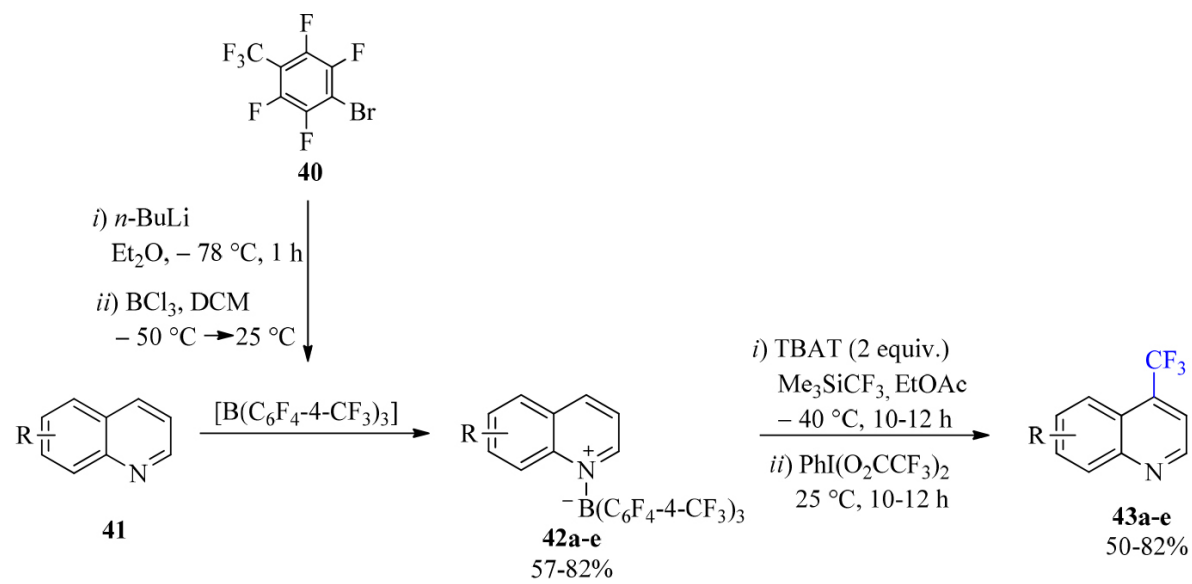

alguns compostos sintetizados<smiles>FC(F)(F)c1ccnc2ccc(/C=C/c3ccccc3)cc12</smiles>

$43 \mathrm{a}$

$69 \%$<smiles>Clc1ccnc2ccc(N3CCOCC3)cc12</smiles>

$43 b$

$59 \%$<smiles>FC(F)(F)c1c(Oc2ccccc2)cnc2ccccc12</smiles>

$43 c$

$70 \%$<smiles>FC(F)(F)c1c(N2CCOCC2)cnc2ccccc12</smiles>

43d<smiles>Clc1c(Br)cnc2ccccc12</smiles>

$43 \mathrm{e}$

Esquema 10. Trifluorometilação de quinolinas

de uma molécula que apresenta atividade de supressão tumoral contra fibrossarcoma (HT1080) e células tumorais pulmonares (A549). ${ }^{93}$ Sendo assim, três etapas reacionais adicionais foram necessárias para o isolamento de 47 em $90 \%$ de rendimento (Esquema 11).

Motati e colaboradores empregaram reações de funcionalização C-H na preparação de uma extensa série de quinolinas 5-substituídas e 5,7-dissubstituídas. Os autores desenvolveram uma metodologia de funcionalização regiosseletiva e livre do uso de metais de transição, empregando várias fontes comerciais de halogênio de baixo custo e fácil acesso, como ácidos tri-halocianúricos. ${ }^{94}$ Nesse trabalho, estas reações foram realizadas à temperatura ambiente e na presença de TXCA ( 0,36 equiv.), sem a necessidade de atmosfera inerte. Vale destacar que a presente metodologia permitiu a obtenção de quinolinas de interesse biológico. Neste contexto, o composto 48 foi empregado na preparação da dicloroquinolina 51, uma substância que atua como agente amiloidogênico. Para isso, TCCA foi inicialmente utilizado na cloração de $\mathbf{4 8}$ e em seguida foi realizada uma etapa de oxidação na presença de $\mathrm{SeO}_{2},{ }^{95}$ fornecendo o produto desejado $51 \mathrm{em} 86 \%$ de rendimento. Adicionalmente, foi realizada a síntese do clioquinol (52), um fármaco com propriedade antifúngica e antiprotozoária. ${ }^{96}$ Desta forma, 49 foi submetido a uma reação de iodação regiosseletiva na presença de NIS como fonte de iodo, em $\mathrm{CHCl}_{3}$ como solvente. Em seguida, clioquinol foi preparado através de uma reação de cloração promovida por TCCA a partir de 49, fornecendo o produto desejado $52 \mathrm{em}$ rendimento moderado. Por último, os autores descreveram a síntese do agente supressor de tumor 47. Nessa síntese, TBCA foi<smiles>[R]C(=O)Nc1cccc2cccnc12</smiles>

44<smiles>[X]N1C(=O)CCC1=O</smiles>

45

$$
\begin{aligned}
& \mathrm{Cu}(\mathrm{OTf})_{2}(10 \mathrm{~mol} \%) \\
& \mathrm{AgNO}_{3} \text { (1 equiv.) } \\
& \underset{\mathrm{KME}, 60{ }^{\circ} \mathrm{C}, 30 \mathrm{~h}}{\stackrel{\mathrm{K}}{\mathrm{DM}}(30 \mathrm{mo})} \\
& {[\mathrm{X}=\mathrm{Br}, \mathrm{I}]}
\end{aligned}
$$<smiles>[R]C(=O)Nc1ccc([X])c2cccnc12</smiles>

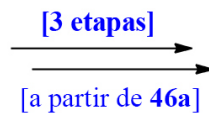

[a partir de 46a]<smiles>O=S(=O)(Nc1ccc(-c2cccnc2)c2cccnc12)c1cccc(C(F)(F)F)c1</smiles><smiles>O=C(Nc1ccc(Br)c2cccnc12)C1CCCC1</smiles><smiles>O=C(Nc1ccc(Br)c2cc(Cl)cnc12)C1CCCC1</smiles>

Esquema 11. Funcionalização C-H de 8-amidoquinolinas 
empregado como fonte de halogênio na bromação da quinolina $\mathbf{5 0}$, em MeCN como solvente. Após a etapa de bromação, o produto halogenado foi submetido à reação de acoplamento na presença do ácido borônico (53) e quantidades catalíticas de paládio. Assim, a quinolina 47 foi preparada em $75 \%$ de rendimento (Esquema 12).

De forma geral, esses protocolos apresentaram alta simplicidade e robustez, fornecendo os produtos de halogenação em rendimentos variando de bons a excelentes, em mais de cem experimentos realizados pelo respectivo grupo de pesquisa.

A reação de Minisci tem sido frequentemente empregada na funcionalização seletiva da ligação $\mathrm{C}-\mathrm{H}$ de heteroarenos. Essa transformação química é caracterizada pela adição de radicais alquílicos a heteroarenos básicos, seguido pela perda formal de um átomo de hidrogênio. ${ }^{97} \mathrm{~A}$ funcionalização $\mathrm{C}-\mathrm{H}$ de anéis quinolínicos com diversos grupos funcionais tem sido alcançada com alta seletividade através da reação de Minisci e suas variantes. Essas reações foram recentemente empregadas na funcionalização seletiva do bosutinibe (54), um fármaco polifuncionalizado que atua como inibidor de tirosina quinase..$^{98}$ Nesse contexto, Shabat e colaboradores desenvolveram uma metodologia sintética de alquilação $\mathrm{C}-\mathrm{H}$ de quinolinas através da utilização de um alquil-sulfinato como fonte de radical alquílico. Nesse trabalho, bosutinibe (54) foi funcionalizado na posição $\mathrm{C} 2$ do anel quinolínico correspondente ao reagir com $\mathbf{5 5}$ na presença de TBHP, TFA e $\mathrm{ZnCl}_{2}$, em DMSO/ $\mathrm{H}_{2} \mathrm{O}$ como solvente. Dessa forma, o produto de reação de difluoroalquilação (56) foi obtido em $67 \%$ de rendimento (Esquema 13). ${ }^{99}$ DiRocco e colaboradores realizaram a ciclopropanação da ligação C-H do bosutinibe (54) através da utilização de um fotocatalisador de irídio. ${ }^{100}$ Para isso, os autores empregaram $2 \mathrm{~mol} \%$ de $\left[\operatorname{Ir}(\mathrm{ppy})_{2}(\mathrm{dtbpy})\right] \mathrm{PF}_{6}(\mathbf{5 7})$ como catalisador na funcionalização $\mathrm{C}-\mathrm{H}$ de $\mathbf{5 4}$, na presença de TFA:MeCN (1:1) como solvente à temperatura ambiente, empregando radiação na região do visível e CPO (58) como fonte de radical ciclopropil. Essa metodologia forneceu o produto de ciclopropanação 59, através da introdução altamente seletiva do grupo ciclopropil na estrutura molecular de 54, em rendimento moderado. Ambos os protocolos empregaram condições reacionais brandas, fornecendo análogos sintéticos de 542 -funcionalizados (56 e 59) com alta regiosseletividade, mesmo na presença de diversos substituintes presentes na estrutura molecular do bosutinibe (54). Com isso, essas metodologias se mostraram como alternativas atraentes na funcionalização tardia (do inglês, late-stage functionalization) ${ }^{101}$ desse fármaco, permitindo o acesso a moléculas não facilmente acessíveis pelas metodologias sintéticas convencionais.

Dependendo da metodologia empregada, as reações de funcionalização $\mathrm{C}-\mathrm{H}$ de quinolinas podem apresentar diferentes regiosseletividades. Esse fato pode ser observado através da funcionalização $\mathrm{C}-\mathrm{H}$ da camptotecina (60), um alcaloide com propriedade antiproliferativa. ${ }^{102}$ Nesse sentido, Zhang e colaboradores realizaram a funcionalização da posição C5 do anel quinolínico da camptotecina (60) através da reação de ativação C-H (Esquema 14). Nesse trabalho, quantidade estequiométrica de $\mathbf{6 1}$ foi utilizada na conversão de 60 na presença de AgOAc e quantidades catalíticas de $\mathrm{Pd}(\mathrm{OAc})_{2}$, em HFIP como solvente. Dessa forma, o produto de alquilação C-H 62 foi obtido em rendimento moderado. ${ }^{103} \mathrm{Em}$

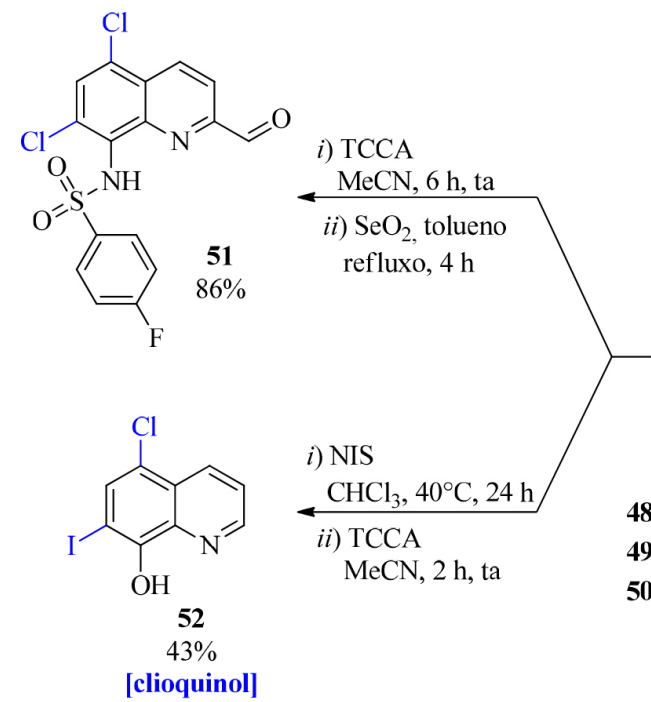

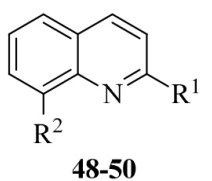

i) $\mathrm{TBCA}$

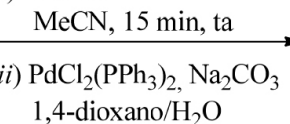

$100{ }^{\circ} \mathrm{C}, 20 \mathrm{~min}, \mu \mathrm{W}$

\begin{abstract}
48: $\mathrm{R}^{1}=\mathrm{CHO}, \mathrm{R}^{2}=p-\mathrm{FSO}_{2} \mathrm{NH}$
49: $\mathrm{R}^{1}=\mathrm{H}, \mathrm{R}^{2}=-\mathrm{OH}$

50: $\left.\mathrm{R}^{1}=\mathrm{H}, \mathrm{R}^{2}=p-\mathrm{FSO}_{2} \mathrm{NH}\right]$
\end{abstract}

$53 \stackrel{1}{\mathrm{O}}$

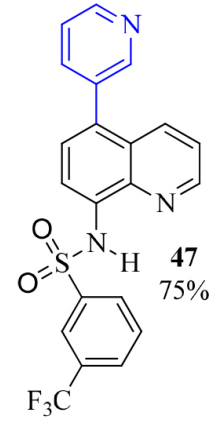

[clioquinol]

Esquema 12. Funcionalização C-H e preparação de quinolinas bioativas

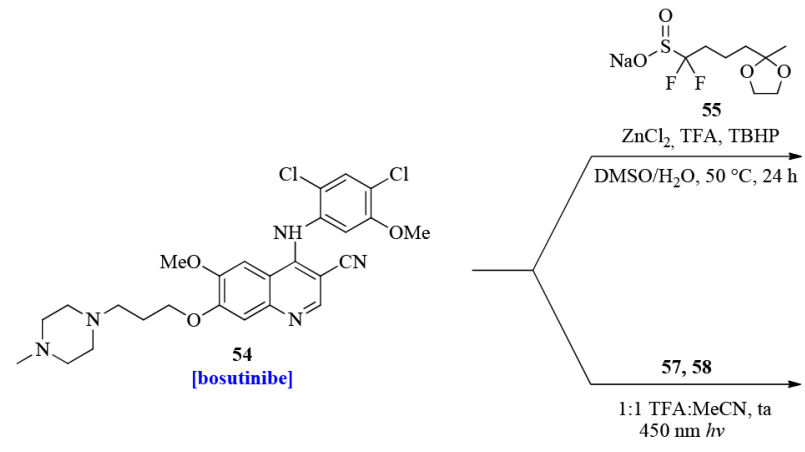

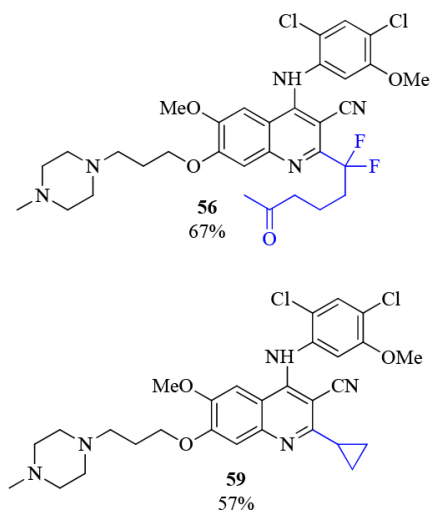
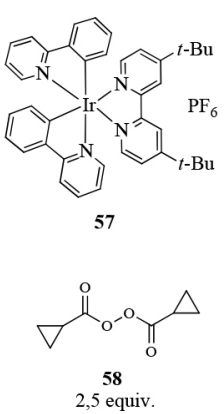

Esquema 13. Funcionalização C-H do bosutinibe (54) 

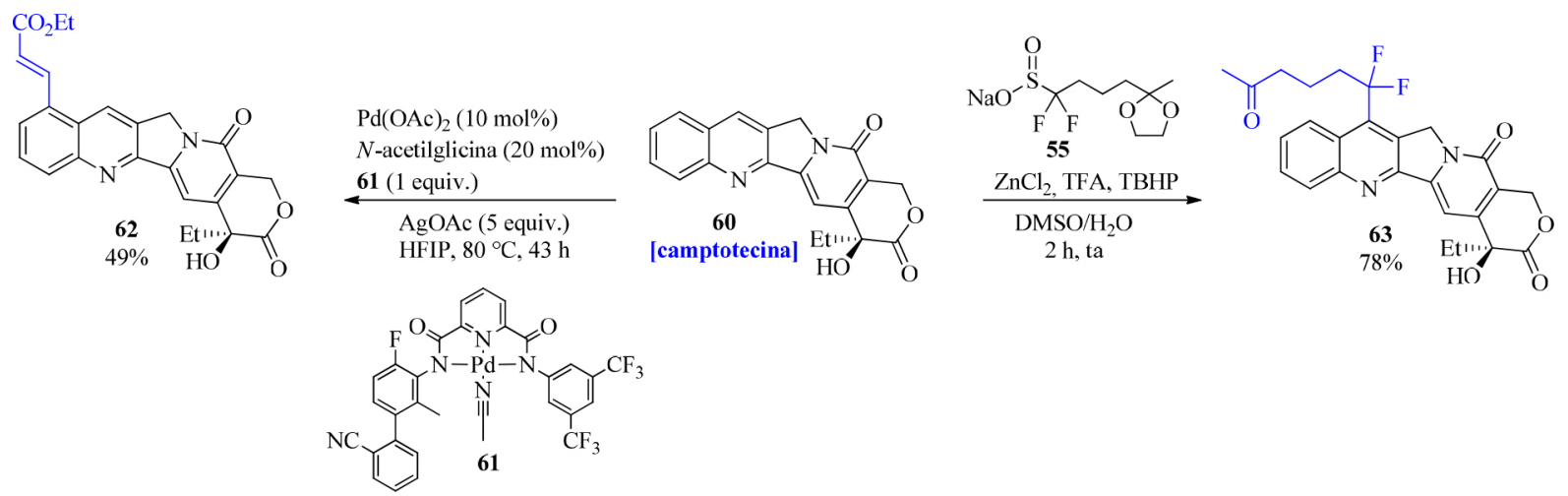

Esquema 14. Regiosseletividade da funcionalização $C$ - $H$ da camptotecina (60)

particular, a funcionalização C-H na posição C5 do anel quinolínico é de fundamental importância na preparação de quinolinas com propriedade antiviral e antiploriferativa (Figura 1). Por outro lado, a funcionalização da posição $\mathrm{C} 4$ foi possível através da reação de alquilação C-H (reação de Minisci) desenvolvida por Shabat e colaboradores. Nesse trabalho, camptotecina (60) reagiu com o sulfinato 55 na presença de TBHP, TFA e $\mathrm{ZnCl}_{2}$, em DMSO/H $\mathrm{H}_{2} \mathrm{O}$ como solvente, fornecendo o produto $\mathbf{6 3} \mathrm{em} 78 \%$ de rendimento, empregando condições reacionais brandas. ${ }^{99}$

\section{Reações de acoplamento}

Reações de acoplamento catalisadas por metais de transição constituem um meio eficiente e direto para formação de novas ligações C-C em química orgânica. ${ }^{104-110}$ Essas transformações químicas têm sido utilizadas na obtenção de diversos compostos heterocíclicos de interesse medicinal e sintético. ${ }^{111,112}$ Em particular, haloquinolinas podem ser funcionalizadas através da reação de acoplamento catalisado por diversos metais de transição. ${ }^{113-121}$ Recentemente, Baenziger e colaboradores empregaram a reação de acoplamento de Suzuki na preparação do dactolisibe (66), um inibidor das quinases PI3K e mTOR. Neste trabalho, $\mathbf{6 6}$ foi obtido em $88 \%$ de rendimento a partir do acoplamento catalisado por paládio entre a quinolina 64 e o ácido borônico 65. (Esquema 15). ${ }^{122}$<smiles>Cn1c(=O)n(-c2ccc(C(C)(C)C#N)cc2)c2c3cc(Br)ccc3ncc21</smiles>

64
$\mathrm{DMF}, \mathrm{H}_{2} \mathrm{O}$
$95-100{ }^{\circ} \mathrm{C}, 2-6 \mathrm{~h}$

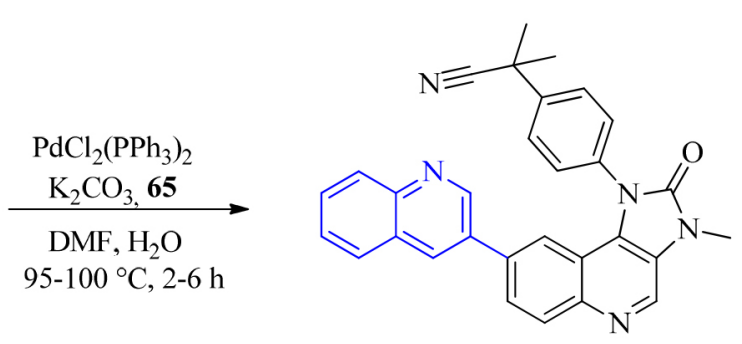

66

$88 \%(152,2 \mathrm{Kg})$

[dactolisibe]
A metodologia desenvolvida pelos autores se mostrou robusta, empregando apenas $0,3 \mathrm{~mol} \%$ de $\mathrm{PdCl}_{2}\left(\mathrm{PPh}_{3}\right)_{2}$ como catalisador. Considerando o risco de contaminação associado ao uso de uma reação catalisada por paládio na última etapa de um processo sintético de um fármaco, ${ }^{123}$ cabe destacar que o escalonamento dessa reação forneceu o dactolisibe (66) em pouco mais de $150 \mathrm{~kg} \mathrm{em}$ massa, com menos de 1 ppm de Pd como contaminante.

Toyota e colaboradores empregaram o acoplamento de Sonogashira catalisado por paládio na obtenção da quinolina 69 , um intermediário na síntese formal do alcaloide natural mappicina (70). ${ }^{124}$ Nessa etapa reacional, a quinolina 67 reagiu com o alquino 68 na presença de quantidades catalíticas de $\mathrm{Pd}\left(\mathrm{PPh}_{3}\right)_{2} \mathrm{Cl}_{2}$ e CuI, em DMF como solvente à temperatura ambiente, em apenas uma hora de reação. Dessa forma, o produto de acoplamento 69 foi obtido em excelente rendimento reacional e empregado posteriormente como intermediário sintético na preparação da mappicina (70), após várias etapas reacionais (Esquema 16).

Adicionalmente, Zhang e colaboradores desenvolveram uma metodologia sintética para a preparação de 8-arilquinolinas funcionalizadas através da sequência reacional composta por borilação-acoplamento de Suzuki-Miyaura. Esse procedimento do tipo one-pot empregou pequenas quantidades de catalisador e ligante ( $1 \mathrm{~mol} \%$ e $3 \mathrm{~mol} \%$, respectivamente) fornecendo os produtos de arilação em apenas uma hora de reação. De forma geral, a metodologia

Esquema 15. Síntese em larga escala do dactolisibe (66) via acoplamento de Suzuki
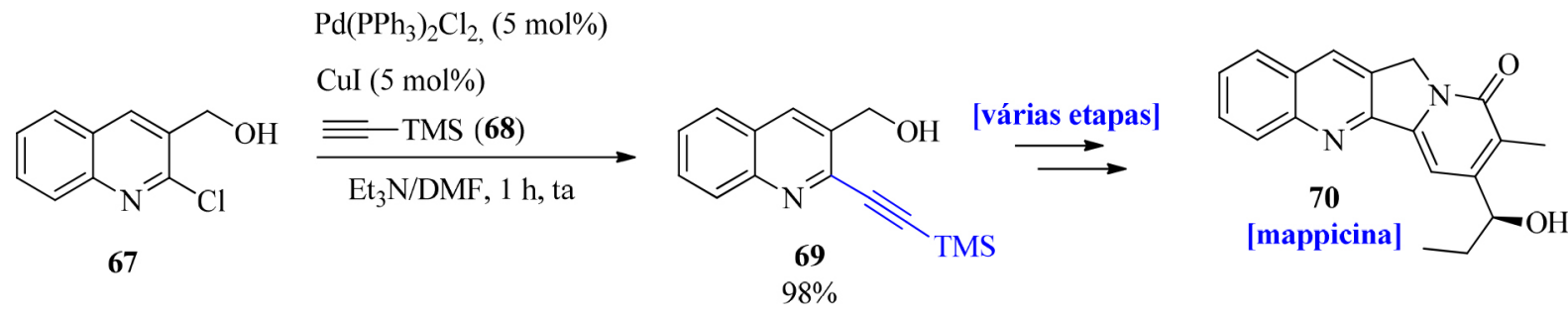

Esquema 16. Preparação da mappicina (70) via acoplamento de Sonogashira 
desenvolvida pelos autores se mostrou robusta, apresentando amplo escopo e alta tolerância a grupos funcionais diversos, fornecendo os produtos de arilação em ótimos rendimentos (Esquema 17). ${ }^{125}$ Esse trabalho permitiu o acesso a 8-arilquinolinas, um núcleo que pode ser empregado como ligante e em importantes estruturas em ciências dos materiais, além de que está presente em diferentes estruturas moleculares de substâncias de interesse biológico.

A diferença de reatividade entre ligações do tipo carbonohalogênio $(\mathrm{C}-\mathrm{X}, \mathrm{X}=\mathrm{F}, \mathrm{Cl}, \mathrm{Br}$ e I) frente às reações de acoplamento tem sido explorada na manipulação quimiosseletiva de derivados quinolínicos. Neste sentido, Billard e colaboradores descreveram a aplicação dessa estratégia na arilação regiosseletiva de quinolinas, através do acoplamento dessulfitativo de cloro-iodo-quinolinas. Nesse trabalho, as quinolinas 75a-d foram sintetizadas a partir de 74a-d na presença de $\mathrm{PhSO}_{2} \mathrm{Na}$ e quantidades catalíticas de $\mathrm{Pd}_{2}(\mathrm{dba})_{3}$ na ordem de 2,5 $\mathrm{mol} \%$, fornecendo os produtos de acoplamento em rendimentos variando de moderados a bons (Esquema 18). ${ }^{126}$

Além da alta seletividade frente aos compostos heteroaromáticos com diferentes substituições, essa metodologia se mostrou como uma alternativa aos reagentes organometálicos tradicionais utilizados nas reações de acoplamento. De forma geral, esse protocolo se mostrou eficiente no acoplamento de 3-iodoquinolinas e o aquecimento empregado nesse procedimento favorece a liberação de $\mathrm{SO}_{2}$ como subproduto de reação.

Sen e colaboradores descreveram a preparação de furo[3,2- $h]$ quinolinas através de uma reação dominó composta por acoplamento de Sonogashira e ciclização entre a di-haloquinolina 76 e um alquino. ${ }^{127}$ Nesse trabalho, uma alquinilação foi inicialmente realizada de forma quimiosseletiva na posição $\mathrm{C} 7$ do anel quinolínico de $\mathbf{7 6}$, na presença de nanopartículas de paládio como catalisador (Pd nps). Essa reação ocorreu na ausência de ligantes, cocatalisador de cobre e solventes orgânicos ou líquidos iônicos e, dessa forma, após formação in situ dos intermediários de reação 77a-e, esses foram ciclizados aos derivados furo[3,2- $h$ ]quinolinas 78a-e correspondentes em rendimentos variando de bons a excelentes. Vale destacar que o catalisador empregado nesse trabalho pôde ser recuperado e reciclado por cinco reações consecutivas sem perda significante de atividade catalítica, ao se investigar os efeitos do reciclo frente a isolidina 79 (Esquema 19).
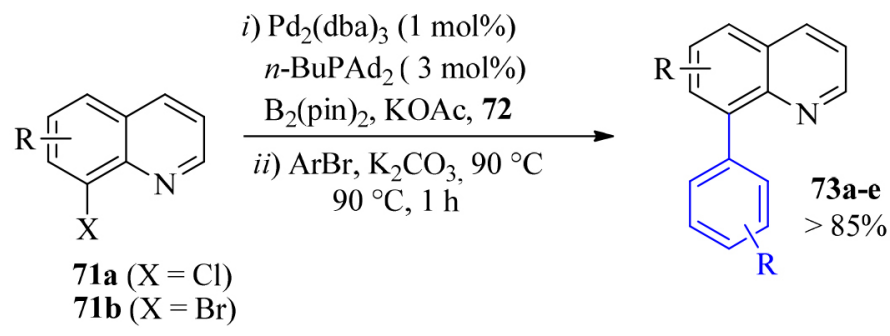<smiles>CC(=O)N(C)C</smiles><smiles>c1ccc(-c2cccc3cccnc23)cc1</smiles><smiles>COc1ccc(-c2cccs2)c2ncccc12</smiles>

$73 \mathrm{e}$ $85 \%$

Esquema 17. Preparação de 8-arilquinolinas via borilação-arilação catalisada por paládio

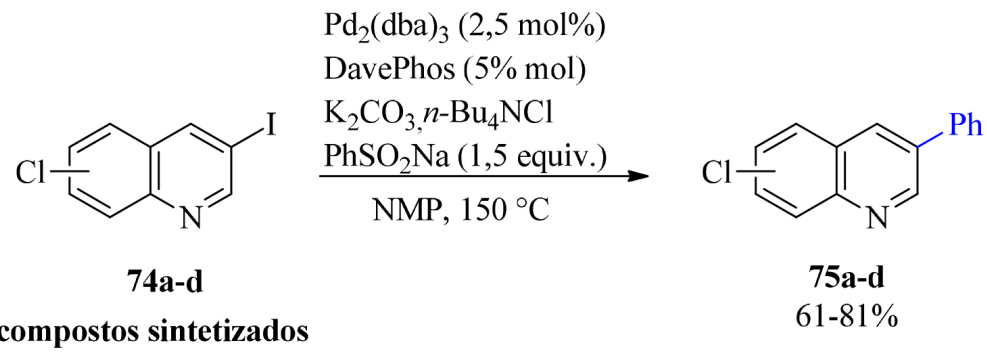

alguns compostos sintetizados<smiles>Clc1cccc2cc(-c3ccccc3)cnc12</smiles>

$75 \mathbf{a}$

$81 \%$<smiles>Clc1ccc2cc(-c3ccccc3)cnc2c1</smiles>

75b

$71 \%$<smiles>Clc1ccc2ncc(-c3ccccc3)cc2c1</smiles>

$60 \%$<smiles>Clc1cccc2ncc(-c3ccccc3)cc12</smiles>

75d

$61 \%$

Esquema 18. Acoplamento dessulfitativo de cloro-iodo-quinolinas 
4,7-Dicloroquinolina (18) foi utilizada como substrato em reações de acoplamento do tipo $s p^{2}-s p^{3}$ catalisadas por ferro com o objetivo de sintetizar quinolinas 4 -funcionalizadas. ${ }^{115}$ Nesse trabalho, Malhotra e colaboradores realizaram a funcionalização quimiosseletiva da 4,7-DCQ (18) através da reação de acoplamento cruzado empregando pequenas quantidades de $\mathrm{Fe}(\mathrm{acac})_{3}$, um catalisador de baixo custo e fácil preparação. Essa reação foi realizada à temperatura ambiente e dispensou o uso de ligantes $\mathrm{e}$ aditivos, fornecendo os produtos de reação desejados (80a-e) em rendimentos variando de moderado a excelentes. Além disso, essa metodologia permitiu a introdução do grupo ciclopropil de forma eficiente. Esse grupo é frequentemente observado na estrutura molecular de diversos candidatos a drogas em estágio clínico e pré-clínico (Esquema 20). ${ }^{128}$

Duplo acoplamento catalisado por paládio foi realizado por Pal e colaboradores durante estudos referentes à dialquinilação da quinolina 81. Inicialmente, os autores realizaram a iodação da 2,4-dicloroquinolina 81 empregando cloreto de acila (82) e NaI, em MeCN como solvente. ${ }^{129}$ Em seguida, o intermediário sintético 83 foi submetido à reação de acoplamento com um alquino, na presença de quantidades catalíticas de $\mathrm{Pd} / \mathrm{C}$ e CuI. Além de empregar catalisadores de baixo custo e fornecer os produtos de duplo acoplamento $(\mathbf{8 4 a}-\mathbf{c})$ em ótimos rendimentos, essa metodologia utilizou água como solvente da reação. Dessa forma, os autores apresentaram uma alternativa sintética atrativa na obtenção de produtos de duplo acoplamento com simplicidade operacional, utilizando reagentes de partida de fácil acesso (Esquema 21).
Há poucos anos, Wolkenberg e colaboradores realizaram a derivatização controlada de quinolinas poli-halogenadas através de sucessivas reações de acoplamento cruzado. ${ }^{130}$ Nesse trabalho, os autores realizaram inicialmente arilação da quinolina 85 na presença de um ácido borônico e quantidades catalíticas de paládio, mesmo na presença de diversas ligações do tipo carbono-halogênio disponíveis. Essa reação permitiu a obtenção da quinolina $\mathbf{8 6}$ com alta seletividade e bom rendimento. Em seguida, condições reacionais similares foram empregadas na preparação quimiosseletiva da quinolina $\mathbf{8 7}$. Esse derivado quinolínico pôde ser preparado devido à diferença de reatividade das ligações $\mathrm{C}$ - $\mathrm{Br}$ e $\mathrm{C}$-Cl. Por último, uma etapa adicional de arilação foi necessária na preparação da quinolina 88. Dessa forma, a reação de acoplamento de Suzuki-Miyaura ocorreu de forma quimiosseletiva na posição C4 do anel quinolínico de $\mathbf{8 7}$, fornecendo o produto desejado $\mathbf{8 8}$ em $73 \%$ de rendimento (Esquema 22).

\section{Reações de substituição aromática}

Reações de substituição nucleofílica aromática são frequentemente empregadas na preparação de diversos derivados quinolínicos de interesse medicinal. ${ }^{131-133}$ De forma geral, essas reações ocorrem nas posições C2 e C4 na porção piridínica do grupo quinolina. ${ }^{134}$ Em 2010, Saidian e colaboradores desenvolveram uma metodologia sintética na preparação de piranoquinolinas, uma classe de derivados quinolínicos que apresenta atividades antialérgica, anti-inflamatória, psicotrópica e estrogênica. ${ }^{135}$ Nesse trabalho, as quinolinas 91a-d foram inicialmente geradas in situ através da condensação de

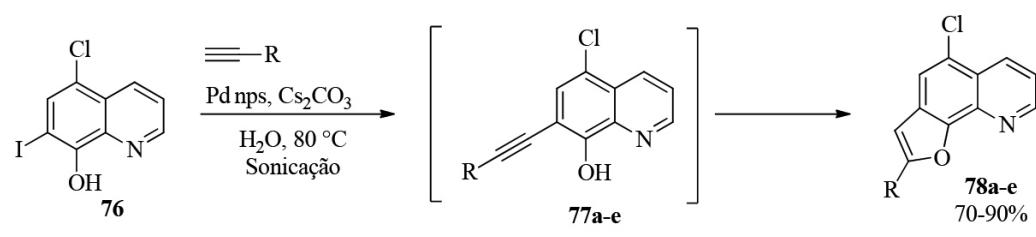

alguns compostos sintetizados
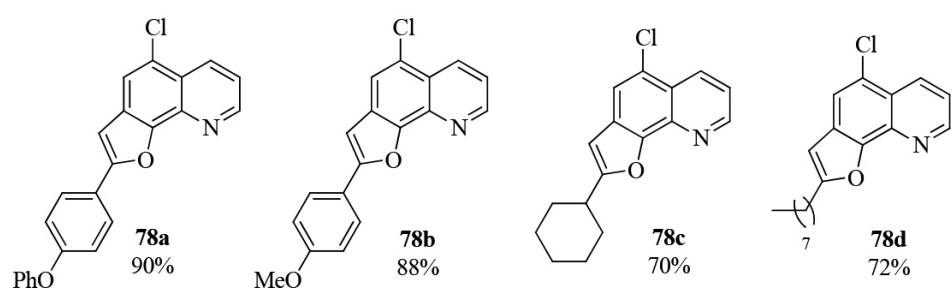
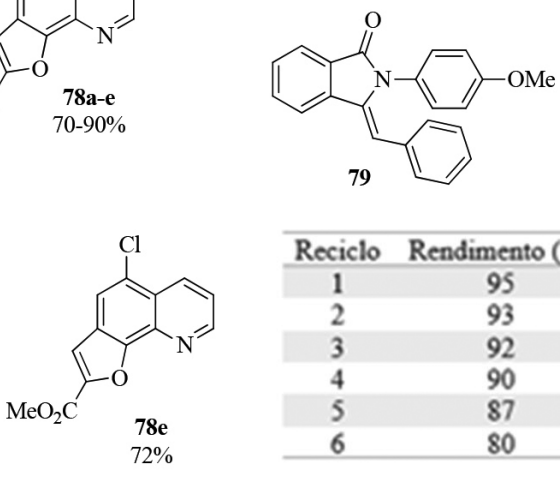

\begin{tabular}{cc}
\hline Reciclo & Rendimento (\%) \\
\hline 1 & 95 \\
2 & 93 \\
3 & 92 \\
4 & 90 \\
5 & 87 \\
6 & 80 \\
\hline
\end{tabular}

Esquema 19. Síntese de furo[3,2-h]quinolinas via acoplamento catalisado por paládio<smiles>Clc1ccc2c(Cl)ccnc2c1</smiles>

18

alguns compostos sintetizados<smiles>Cc1ccnc2cc(Cl)ccc12</smiles>

$80 a$

$96 \%$<smiles>Clc1ccc2c(C3CC3)ccnc2c1</smiles>

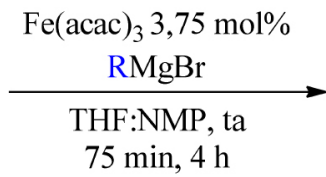

$75 \mathrm{~min}, 4 \mathrm{~h}$<smiles>[R]c1ccnc2cc([O+])ccc12</smiles>

$60-96 \%$<smiles>Clc1ccc2c(CCC3OCCCO3)ccnc2c1</smiles><smiles>CCCc1ccnc2cc(Cl)ccc12</smiles><smiles>Clc1ccc2c(C3CCCCC3)ccnc2c1</smiles>

Esquema 20. Alquilação regiosseletiva de 4,7-dicloroquinolina (18) 


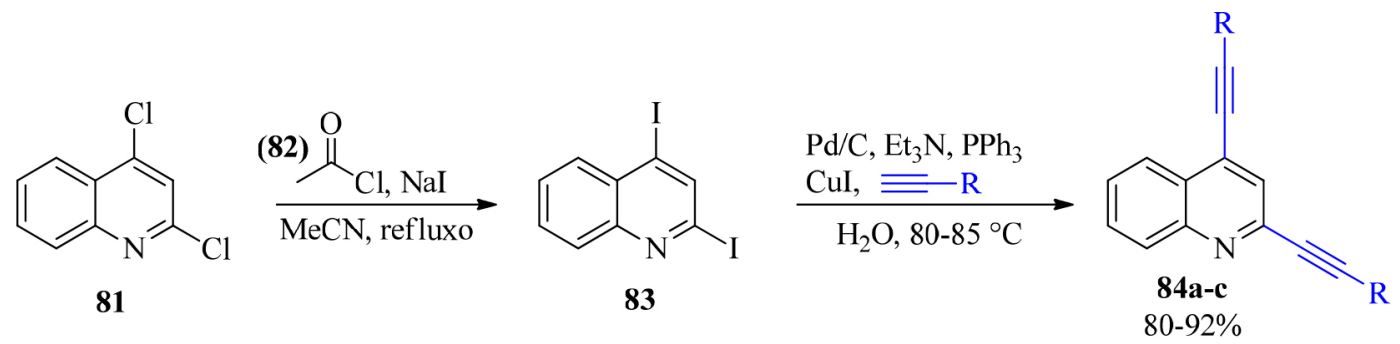

\section{alguns compostos sintetizados}<smiles>C(#Cc1cc(C#Cc2ccccc2)c2ccccc2n1)c1ccccc1</smiles>

84a $85 \%$<smiles>OC1(C#Cc2cc(C#CC3(O)CCCCC3)c3ccccc3n2)CCCCC1</smiles>

84b

$92 \%$<smiles>OCCCC#Cc1cc(C#CCCCO)c2ccccc2n1</smiles>

$84 \mathrm{c}$ $80 \%$

Esquema 21. Quinolinas preparadas por duplo acoplamento catalisado por paládio<smiles>Clc1cc2ncc(Br)c(Cl)c2cc1I</smiles>

85

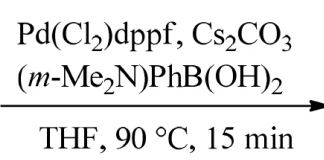<smiles>CN(C)c1cccc(-c2cc3c(Cl)c(Br)cnc3cc2Cl)c1</smiles>

$\mathrm{Pd}\left(\mathrm{Cl}_{2}\right) \mathrm{dppf}, \mathrm{Cs}_{2} \mathrm{CO}_{3}$ $(p-\mathrm{Me}) \mathrm{PhB}(\mathrm{OH})_{2}$

THF, $160^{\circ} \mathrm{C}, 15 \mathrm{~min}$<smiles>Cc1ccc(-c2cnc3cc(Cl)c(-c4cccc(N(C)C)c4)cc3c2Cl)cc1</smiles>

$\mathrm{Pd}\left(\mathrm{Cl}_{2}\right) \mathrm{dppf}, \mathrm{Cs}_{2} \mathrm{CO}_{3}$ $\mathrm{PhB}\left(\mathrm{C}_{5} \mathrm{H}_{10} \mathrm{O}_{2}\right)$

$\mathrm{THF}, 160^{\circ} \mathrm{C}, 30 \mathrm{~min}$<smiles>Cc1ccc(-c2cnc3cc(Cl)c(-c4cccc(N(C)C)c4)cc3c2-c2ccccc2)cc1</smiles>

88

$73 \%$

Esquema 22. Arilação controlada da quinolina poli-halogenada 85

Knoevenagel a partir de 2-cloroquinolinas 89a-d, empregando

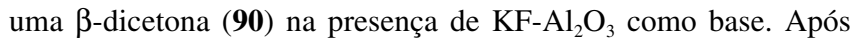
condensação, esses intermediários (91a-d) sofreram uma reação de substituição nucleofílica intramolecular levando à formação das piranoquinolinas 92a-d, em rendimentos moderados (Esquema 23).

Reações de aminação são importantes transformações químicas na obtenção de compostos quinolínicos nitrogenados biologicamente ativos. ${ }^{136-138}$ Essa reação permite a funcionalização do grupo quinolínico através da formação de ligação do tipo $\mathrm{C}-\mathrm{N}$, podendo ser empregada na síntese de importantes fármacos como a amodiaquina e a cloroquina. ${ }^{139}$ Nesse sentido, Katti e colaboradores empregaram reações de substituição nucleofílica na preparação de diversos análogos sintéticos da cloroquina, uma substância com propriedade antimalárica (Esquema 24 - condição 1). ${ }^{140}$

Além da preparação de vários análogos enantiomericamente puros da cloroquina, esse trabalho descreveu a síntese de ambos enantiômeros desta substância (95a-b). De forma geral, esses derivados de quinolina foram preparados a partir da reação entre 4,7-DCQ (18) e 94 em fenol como solvente, com rendimentos moderados. Alternativamente, Clososki e colaboradores realizaram a preparação de um análogo sintético da cloroquina (95c), empregando condições reacionais verdes. Nesta metodologia, a reação foi realizada sob micro-ondas, utilizando glicerina como solvente, fornecendo assim o produto desejado $\mathbf{9 5 c}$ em $65 \%$ de rendimento (Esquema 24 - condição 2). ${ }^{73}$

Reações de substituição eletrofílica também podem ser empregadas na funcionalização de quinolinas. Essas transformações químicas são mediadas por um ácido de Lewis e ocorrem comumente na porção carbocíclica do anel quinolínico, mais precisamente nas posições C5 e C8. ${ }^{141-145}$ Billard e colaboradores empregaram uma reação de substituição eletrofílica na preparação da quinolina 97. Nesse trabalho, a cloroquinolina 96 foi submetida inicialmente a uma reação de heteroacoplamento catalisado por paládio. Em seguida, a reação de substituição eletrofílica foi realizada empregando NIS como fonte de iodo em $\mathrm{AcOH}$ como solvente, fornecendo o produto desejado halogenado em C5 (97), em 73\% de rendimento. ${ }^{146}$ Adicionalmente, quando a quinolina 96 foi submetida à reação de substituição eletrofílica sob as mesmas condições reacionais, o 


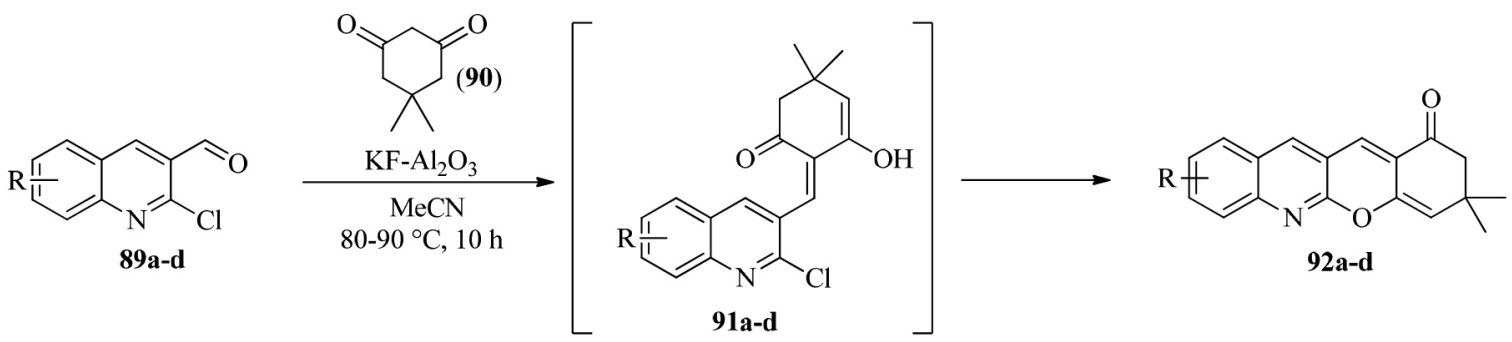

alguns compostos sintetizados
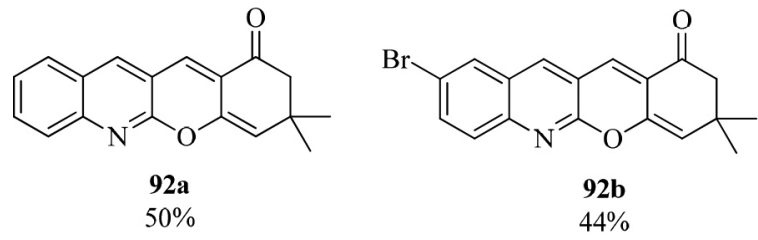<smiles>COc1ccc2cc3c(nc2c1)OC1=CC(C)(C)CC(=O)C1=C3</smiles><smiles>Cc1cc(C)c2cc3c(nc2c1)OC1=CC(C)(C)CC(=O)C1=C3</smiles>

Esquema 23. Sintese de piranoquinolinas 92a-d<smiles>[R]c1cnc2cc(Cl)ccc2c1Cl</smiles>

\section{[condição 1]}

94, fenol

$\frac{140^{\circ} \mathrm{C}, 12 \mathrm{~h}}{\text { [condição 2] }}$

94, glicerina, $\mu \mathrm{W}$

$150{ }^{\circ} \mathrm{C}, 4 \mathrm{~h}$<smiles>[R]c1cnc2cc(Cl)ccc2c1NC(C)CCCN(CC)CC</smiles><smiles>CCN(CC)CCCC(C)N</smiles>

alguns compostos sintetizados

[condição I]<smiles>CCN(CC)CCC[C@H](C)Nc1ccnc2cc(Cl)ccc12</smiles>

[(S)-cloroquina]<smiles>CCN(CC)CCC[C@H](C)Nc1ccnc2cc(Cl)ccc12</smiles>

[condição 2$]$<smiles>CCN(CC)CCCC(C)Nc1c(Cl)cnc2cc(Cl)ccc12</smiles>

Esquema 24. Aminação de quinolinas halogenadas

produto de iodação em C3 (98) foi obtido como único regioisômero, em ótimo rendimento (Esquema 25).

\section{Funcionalização seletiva de quinolinas sob fluxo contínuo}

Nos últimos anos, diferentes protocolos reacionais têm sido executados sob regime de fluxo contínuo por meio do uso de microrreatores, os quais apresentam diâmetros reduzidos e proporcionam um melhor ambiente reacional, uma vez que as transferências de massa e calor se processam de forma otimizada. ${ }^{147-149}$ Além disso, a química em fluxo tem se destacado como uma alternativa interessante para a realização de reações de maneira segura, rápida, escalonável, com menor custo e maior economia atômica. ${ }^{150,151}$ Nesse contexto, algumas metodologias clássicas para a síntese de quinolinas substituídas como as reações de Friedländer, ConradLimpach e Doebner-Miller, bem como as reações de cicloadição, foram realizadas com êxito sob condições de fluxo contínuo. ${ }^{152-156} \mathrm{~A}$ funcionalização do núcleo quinolínico foi demonstrada no trabalho de Lindhardt e colaboradores, por meio do uso de dois reatores tubulares para a $N$-alquilação e $\alpha$-tri-halometilação de quinolinas e isoquinolinas, com a vantagem de que as últimas puderam ser escalonadas para a produção de $94 \mathrm{mmol} / \mathrm{h}$ com o tempo de residência de 9 min (Esquema 26)..$^{157}$

Por fim, vale destacar a cloração de quinolinas sob condições de fluxo contínuo, devido à grande importância medicinal dessa classe de compostos. ${ }^{158}$ Recentemente, Guo e colaboradores desenvolveram uma metodologia sustentável para a síntese de 2-cloroquinolinas substituídas por meio de intermediários fluorados em reatores de fluxo contínuo (Esquema 27). ${ }^{159}$ Nesse trabalho, os autores compararam os processos em batelada e em fluxo contínuo para a cloração da quinolina não substituída e observaram que executando a reação em balão reacional convencional foi possível obter um rendimento máximo de $34 \%$ do produto (2-cloroquinolina) após 8 horas de reação, ao passo que a utilização de um sistema de fluxo contínuo para a mesma reação, equipado com um reator tubular de $6 \mathrm{~mL}$ e com bombas peristálticas configuradas para um bombeamento das soluções reagentes na taxa de $0,1 \mathrm{~mL} \mathrm{~min}^{-1}$, viabilizou um rendimento reacional de $87 \%$ após 30 minutos de reação. Dessa forma, o uso de microreatores possibilitou um melhor rendimento e um menor tempo reacional quando comparado ao processo em batelada.

De forma geral, ambas metodologias se mostraram eficientes na funcionalização do núcleo quinolínico, evidenciando a importância 
<smiles>CN1CCN(c2ccc(I)c3cccnc23)CC1</smiles>

i) $\mathrm{Pd}_{2}(\mathrm{dba})_{3}(5 \%),{ }^{\mathrm{t}} \mathrm{BuONa}$

DavePhos (10\%)

$N$-metilpiperazina

1,4-dioxano, $110^{\circ} \mathrm{C}$

ii) NIS, $\mathrm{AcOH}, 80^{\circ} \mathrm{C}$<smiles>Clc1cccc2cccnc12</smiles><smiles>CC(C)(C)[Mg]O</smiles><smiles>Clc1cccc2cc(I)cnc12</smiles>

Esquema 25. Funcionalização de quinolinas via substituição eletrofílica

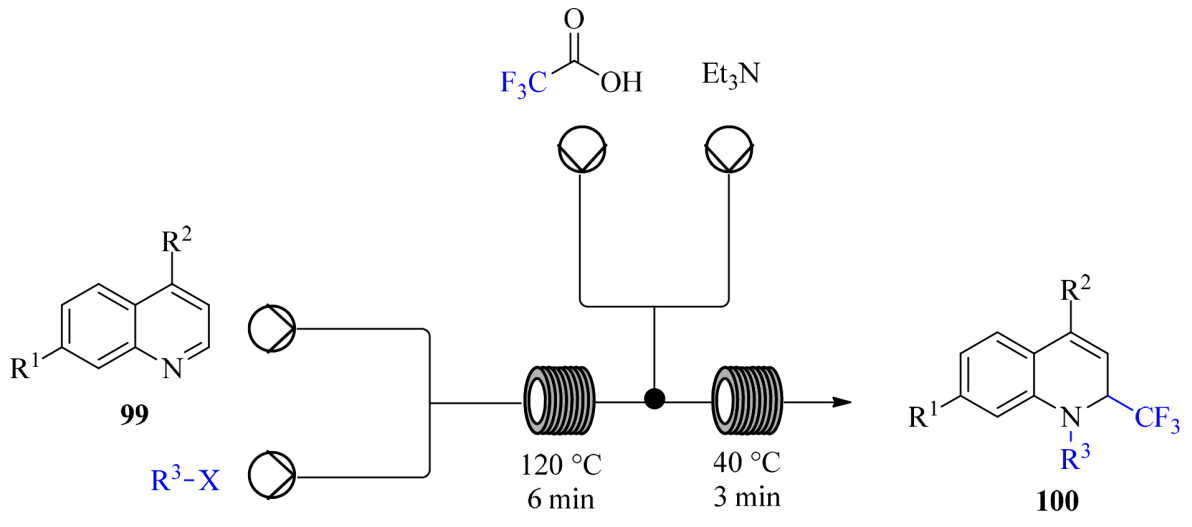

$\left[\mathrm{R}^{1}=\mathrm{H}, \mathrm{Cl} . \mathrm{R}^{2}=\mathrm{H}, \mathrm{Me}\right.$, OCyp. $\mathrm{R}^{3}=\mathrm{Me}, i$-Pr. $\mathrm{X}=\mathrm{I}$, OTs $]$

Esquema 26. Tri-halometilação de quinolinas em fluxo contínuo<smiles>[R][I-][N+]1([18F])CC[N+]([B-])(CCl)CC1</smiles>

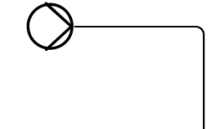

102<smiles>CC1(C)C(=O)N(Cl)C(=O)N1Cl</smiles><smiles>C1=CCCC1</smiles>

103

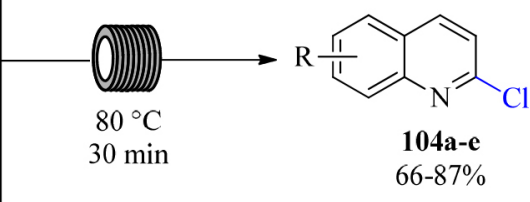

alguns compostos sintetizados<smiles>Clc1ccc2ccccc2n1</smiles>

104a $87 \%$<smiles>Cc1ccc2nc(Cl)ccc2c1</smiles>

104b<smiles>Clc1nc2ccccc2cc1Br</smiles>

$104 \mathrm{c}$<smiles>Clc1ccc2ccc3ccccc3c2n1</smiles>

$66 \%$<smiles>COC(=O)c1ccc2nc(Cl)ccc2c1</smiles>

Esquema 27. Síntese de 2-cloroquinolinas substituídas em fluxo contínuo

deste tipo de processo na preparação de quinolinas com alta diversidade estrutural, alta seletividade e bons rendimentos.

\section{CONCLUSÕES}

Nos últimos 20 anos, o desenvolvimento de bases metálicas mistas, tais como amidetos de magnésio e zinco, tem contribuído para significantes avanços na metalação dirigida de quinolinas, permitindo a síntese de derivados funcionalizados com alta regiosseletividade, através da reação dos intermediários organometálicos correspondentes com diferentes eletrófilos. Adicionalmente, a formação de ligação carbono-metal em quinolinas pode ser alcançada através das reações de troca halogênio-metal, a partir de haloquinolinas. Embora diversos reagentes organometálicos estejam disponíveis comercialmente para essa finalidade, iPrMgCl$\cdot \mathrm{LiCl}$ tem sido empregado como reagente de escolha nesse tipo de reação. Vale destacar que a combinação das reações de troca halogênio-metal e de metalação dirigida pode ser aplicada eficientemente na preparação seletiva de derivados quinolínicos de interesse medicinal. De forma alternativa, as reações de funcionalização C-H têm sido amplamente 
empregadas na preparação de anéis quinolínicos funcionalizados com alta compatibilidade a grupos funcionais diversos e condições reacionais brandas, permitindo a modificação seletiva de ligações do tipo C-H relativamente inertes, mesmo em compostos quinolínicos altamente funcionalizados. Além dessas transformações químicas mencionadas anteriormente, as reações de acoplamento catalisado por metais de transição têm sido utilizadas de forma eficiente na modificação química de haloquinolinas estruturalmente diversas. Essa reação permite a síntese de derivados quinolínicos quimicamente modificados através da formação de novas ligações químicas do tipo C-C. Além disso, as reações de substituição aromática têm sido largamente utilizadas na preparação de derivados quinolínicos funcionalizados, sobretudo no campo da química medicinal. Do ponto de vista operacional, as reações em fluxo têm se mostrado uma atraente alternativa na funcionalização de anéis quinolínicos. Esses processos permitem realizar o escalonamento dessas reações com rapidez, segurança e alta economia atômica, sendo cada vez mais empregados como uma alternativa sintética ambientalmente amigável. Em suma, todas as estratégias mencionadas acima formam um conjunto de ferramentas sintéticas fundamentais na funcionalização seletiva de quinolinas, permitindo o acesso a quimiotecas com alta diversidade estrutural. Esse fato é de fundamental importância ao se considerar a aplicação sintética desse núcleo, sobretudo na preparação de moléculas de interesse comercial e medicinal.

\section{AGRADECIMENTOS}

Os autores agradecem ao ministério da Saúde, Conselho Nacional de Desenvolvimento Científico e Tecnológico (CNPq, processo no 140137/2018-1), Coordenação de Aperfeiçoamento de Pessoal de Nível Superior (CAPES, finance code 001) e ao apoio financeiro da Fundação de Amparo à Pesquisa do Estado de São Paulo - Brasil (FAPESP - Processos: 2015/21364-6, 2018/14150-8, e 2019/05838-9). Os autores declaram não haver conflitos de interesse.

\section{REFERÊNCIAS}

1. Clososki, G. C.; Soldi, R. A.; da Silva, R. M.; Guaratini, T.; Lopes, J. N. C.; Pereira, P. R. R.; Lopes, J. L. C.; dos Santos, T.; Martins, R. B.; Costa, C. S.; de Carvalho, A. N.; da Silva, L. L. P.; Arruda, E.; Lopes, N. P.; J. Braz. Chem. Soc. 2020, 31, 1552.

2. dos Santos, T.; Grundke, C.; Lucas, T.; Großmann, L.; Clososki, G. C.; Opatz, T.; Eur. J. Org. Chem. (2020), doi:10.1002/ejoc.202000970.

3. Dua, R.; Shrivastava, S.; Sonwane, S. K.; Srivastava, S. K.; Adv. Biol. Res. 2011, 5, 120 .

4. Vitaku, E.; Smith, D. T.; Njardarson, J. T.; J. Med. Chem. 2014, 57, 10257.

5. Chung, P. Y.; Bian, Z. X.; Pun, H. Y.; Chan, D.; Chan, A. S. C.; Chui, C. H.; Tang, J. C. O.; Lam, K. H.; Future Med. Chem. 2015, 7, 947.

6. Da Silva, L. E.; De Sousa, P. T.; Joussef, A. C.; Piovezan, C.; Neves, A.; Quim. Nova 2008, 31, 1161 .

7. Margariti, A.; Papakonstantinou, V. D.; Stamatakis, G. M.; Demopoulos, C. A.; Schnakenburg, G.; Andreopoulou, A. K.; Giannopoulos, P.; Kallitsis, J. K.; Philippopoulos, A. I.; Polyhedron 2020, 178, 114336.

8. Cao, X.; Ding, Q.; Gao, A.; Li, Y.; Chang, X.; Wu, Y.; New J. Chem. 2018, 42, 6305 .

9. Song, H.; Zhang, Z.; Dyes Pigm. 2019, 165, 172.

10. Dai, Y.; Liu, X.; Wang, P.; Fu, J.; Yao, K.; Xu, K.; RSC Adv. 2016, 6, 99933

11. Li, J. Y.; Chen, C. Y.; Ho, W. C.; Chen, S. H.; Wu, C. G.; Org. Lett. 2012, 14,5420 .

12. Kaya, İ.; Er, G.; Temizkan, K.; Polym. Bull. 2018, 75, 1809.
13. Isakova, A.; Efremova, O.; Pullan, N.; Lüer, L.; Topham, P. D.; RSC Adv. 2016, 6, 6598.

14. Xu, C.; Han, Y.; Xu, S.; Wang, R.; Yue, M.; Tian, Y.; Li, X.; Zhao, Y.; Gong, P.; Eur. J. Med. Chem. 2020, 186, 111867.

15. Kala, P.; Sharif, S. K.; Murali Krishna, C.; Ramachandran, D.; Med. Chem. Res. 2020, 29, 136.

16. Jin, G.; Xiao, F.; Li, Z.; Qi, X.; Zhao, L.; Sun, X.; ChemMedChem 2020, 15,600 .

17. Chen, T.; Zhuo, L. S.; Liu, P. F.; Fang, W. R.; Li, Y. M.; Huang, W.; Eur. J. Med. Chem. 2020, 192, 112174.

18. Nan, X.; Li, H. J.; Fang, S. B.; Li, Q. Y.; Wu, Y. C.; Eur. J. Med. Chem. 2020, 193, 112241.

19. Al-Sanea, M. M.; Elkamhawy, A.; Paik, S.; Lee, K.; El Kerdawy, A. M.; Abbas, B. S. N.; Roh, E. J.; Eldehna, W. M.; Elshemy, H. A. H.; Bakr, R. B.; Farahat, I. A.; Alzarea, A. I.; Alzarea, S. I.; Alharbi, K. S.; Abdelgawad, M. A.; Bioorg. Med. Chem. 2020, 28, 115525.

20. Khelifi, I.; Pecnard, S.; Bernadat, G.; Bignon, J.; Levaique, H.; Dubois, J.; Provot, O.; Alami, M.; ChemMedChem 2020, 15, 1571.

21. Singh, H.; Nand, B.; Sindhu, J.; Khurana, J. M.; Sharma, C.; Aneja, K. R.; J. Braz. Chem. Soc. 2014, 25, 1178.

22. Calvert, M. B.; Furkert, D. P.; Cooper, C. B.; Brimble, M. A.; Bioorg. Med. Chem. Lett. 2020, 30, 127172.

23. Bhat, S. Y.; Jagruthi, P.; Srinivas, A.; Arifuddin, M.; Qureshi, I. A.; Eur. J. Med. Chem. 2020, 186, 111860.

24. Li, F.; Lee, E. M.; Sun, X.; Wang, D.; Tang, H.; Zhou, G. C.; Eur. J. Med. Chem. 2020, 187, 111925.

25. Tang, Q.; Xu, Z.; Jin, M.; Shu, T.; Chen, Y.; Feng, L.; Zhang, Q.; Lan, K.; Wu, S.; Zhou, H. B.; Eur. J. Med. Chem. 2020, 202, 112310.

26. Saul, S.; Pu, S. Y.; Zuercher, W. J.; Einav, S.; Asquith, C. R. M.; Bioorg. Med. Chem. Lett. 2020, 30, 127284.

27. Muscia, G. C.; Pacheco, F. J. R.; Asís, S. E.; Buldain, G. Y.; Frank, F. M.; Eur. J. Med. Chem. 2020, 186, 111877.

28. El-Azzouny, A. M. A. E. S.; Aboul-Enein, M. N.; Hamissa, M. F.; Drug Dev. Res. (2020), doi: 10.1002/ddr.21678.

29. Walle, T. V.; Boone, M.; Puyvelde, J. V.; Combrinck, J.; Smith, P. J.; Chibale, K.; Mangelinckx, S.; D'hooghe, M.; Eur. J. Med. Chem. 2020, 198, 112330.

30. Silva, T. L.; Bartolomeu, A. A.; de Jesus, H. C. R.; de Oliveira, K. T.; Fernandes, J. B.; Brömme, D.; Vieira, P. C.; J. Braz. Chem. Soc. 2020, 31,1605 .

31. Aly, M. R. E.; Nawwar, G. A. M.; El Mageed, A. E. M. A.; El Kafafy, A. K. M. A.; Afinidad 2010, 67, 310 .

32. Rosado-Solano, D. N.; Barón-Rodríguez, M. A.; Sanabria Florez, P. L.; Luna-Parada, L. K.; Puerto-Galvis, C. E.; Zorro-González, A. F.; Kouznetsov, V. V.; Vargas-Méndez, L. Y.; J. Agric. Food Chem. 2019, 67, 9210.

33. Chanzá, N. M.; Xie, W.; Bilen, M. A.; Dzimitrowicz, H.; Burkart, J.; Geynisman, D. M.; Balakrishnan, A.; Bowman, I. A.; Jain, R.; Stadler, W.; Zakharia, Y.; Narayan, V.; Beuselinck, B.; McKay, R. R.; Tripathi, A.; Pachynski, R.; Hahn, A. W.; Hsu, J.; Shah, S. A.; Lam, E. T.; Rose, T. L.; Mega, A. E.; Vogelzang, N.; Harrison, M. R.; Mortazavi, A.; Plimack, E. R.; Vaishampayan, U.; Hammers, H.; George, S.; Haas, N.; Agarwal, N.; Pal, S. K.; Srinivas, S.; Carneiro, B. A.; Heng, D. Y. C.; Bosse, D.; Choueiri, T. K.; Harshman, L. C.; Lancet Oncol. 2019, 20 , 581.

34. Capozzi, M.; Divitiis, C. D.; Ottaiano, A.; Arx, C. V.; Scala, S.; Tatangelo, F.; Delrio, P.; Tafuto, S.; Cancer Manag. Res. 2019, 11, 3847.

35. Torres, R. E. M.; Banegas, E. I.; Mendoza, M.; Diaz, C.; Bucheli, S. T. M.; Fontecha, G. A.; Alam, M. T.; Goldman, I.; Udhayakumar, V.; Zambrano, J. O. N.; Am. J. Trop. Med. Hyg. 2013, 88, 850.

36. Graydon, I.; Beatty, E.; Paul, S.; Us, M. N.; Hauck, J. A.; US6664397B1, 2006. 
37. Novitt-Moreno, A.; Ransom, J.; Dow, G.; Smith, B.; Read, L. T.; Toovey, S.; Travel Med. Infect. Dis. 2017, 17, 19.

38. Paggiaro, P.; Bacci, E.; Ther. Adv. Chronic Dis. 2011, 2, 47.

39. Stephens, D. E.; Larionov, O. V.; Tetrahedron 2015, 71, 8683.

40. Iwai, T.; Sawamura, M.; ACS Catal. 2015, 5, 5031.

41. Wang, D.; Désaubry, L.; Li, G.; Huang, M.; Zheng, S.; Adv. Synth. Catal. (2020), doi: 10.1002/adsc.202000910.

42. Dhiman, A. K.; Thakur, A.; Kumar, R.; Sharma, U.; Asian J. Org. Chem. (2020), doi: 10.1002/ajoc.202000341.

43. Mulvey, R. E.; Mongin, F.; Uchiyama, M.; Kondo, Y.; Angew. Chem., Int. Ed. 2007, 46, 3802.

44. Snieckus, V.; Chem. Rev. 1990, 90, 879.

45. Metallinos, Costa.; Stromski, K.; Top. Heterocycl. Chem. 2013, 31, 65.

46. Haag, B.; Mosrin, M.; Ila, H.; Malakhov, V.; Knochel, P.; Angew. Chem., Int. Ed. 2011, 50, 9794.

47. Souza, M, V, N.; Almeida, M. V.; Quim. Nova 2002, 25, 89.

48. Turck, A.; Tetrahedron 2001, 57, 4489.

49. Queguiner, G.; Marsais, F.; Snieckus, V.; Epsztajn, J.; Adv. Heterocycl. Chem. 1991, 52, 187.

50. Harms, A. E.; Org. Process Res. Dev. 2004, 8, 666.

51. Seggio, A.; Chevallier, F.; Vaultier, M.; Mongin, F.; J. Org. Chem. 2007, 72,6602 .

52. Fur, N. Le; Mojovic, L.; Plé, N.; Turck, A.; Marsais, F.; Tetrahedron 2005, 61, 8924.

53. Schlosser, M.; Marull, M.; Eur. J. Org. Chem. 2003, 8, 1569.

54. Rohbogner, C. J.; Clososki, G. C.; Knochel, P.; Angew. Chem., Int. Ed. 2008, 47, 1503.

55. Clososki, G. C.; Rohbogner, C. J.; Knochel, P.; Angew. Chem., Int. Ed. 2007, 46, 7681.

56. Dong, Z.; Clososki, G. C.; Wunderlich, S. H.; Unsinn, A.; Li, J.; Knochel, P.; Chem. - Eur. J. 2009, 15, 457.

57. Batista, J. H. C.; Santos, F. M. D.; Bozzini, L. A.; Vessecchi, R.; Oliveira, A. R. M.; Clososki, G. C.; Eur. J. Org. Chem. 2015, $2015,967$.

58. Amaral, M. F. Z. J.; Baumgartner, A. A.; Vessecchi, R.; Clososki, G. C.; Org. Lett. 2015, 17, 238.

59. Haas, D.; Mosrin, M.; Knochel, P.; Org. Lett. 2013, 15, 6162.

60. Klatt, T.; Markiewicz, J. T.; Sämann, C.; Knochel, P.; J. Org. Chem. 2014, 79, 4253.

61. Noji, T.; Fujiwara, H.; Okano, K.; Tokuyama, H.; Org. Lett. 2013, 15, 1946.

62. Klatt, T.; Roman, D. S.; León, T.; Knochel, P.; Org. Lett. 2014, 16, 1232.

63. Unsinn, A.; Rohbogner, C. J.; Knochel, P.; Adv. Synth. Catal. 2013, 355, 1553.

64. Monzõn, G.; Tirotta, I.; Knochel, P.; Angew. Chem., Int. Ed. 2012, 51, 10624.

65. De Oliveira, A. R. M.; Szczerbowski, D.; Quim. Nova 2009, 32, 1971.

66. Jaric, M.; Haag, B. A.; Manolikakes, S. M.; Knochel, P.; Org. Lett. 2011, 13, 2306.

67. Dos Santos, F. M.; Batista, J. H. C.; Vessecchi, R.; Clososki, G. C.; Synlett 2015, 26, 2795.

68. Bertallo, C. R. d. S.; Arroio, T. R.; Toledo, M. F. Z. J.; Sadler, S. A.; Vessecchi, R.; Steel, P. G.; Clososki, G. C.; Eur. J. Org. Chem. 2019, 2019, 5205.

69. Balkenhohl, M.; Heinz, B.; Abegg, T.; Knochel, P.; Org. Lett. 2018, 20, 8057.

70. Rohbogner, C. J.; Wirth, S.; Knochel, P.; Org. Lett. 2010, 12, 1984.

71. Elliott, J. M.; Carling, R. W.; Chambers, M.; Chicchi, G. G.; Hutson, P. H.; Jones, A. B.; MacLeod, A.; Marwood, R.; Meneses-Lorente, G.; Mezzogori, E.; Murray, F.; Rigby, M.; Royo, I.; Russell, M. G. N.; Sohal, B.; Tsao, K. L.; Williams, B.; Bioorg. Med. Chem. Lett. 2006, $16,5748$.

72. Jeong, H. J.; Chae, S.; Jeong, K.; Namgoong, S. K.; Eur. J. Org. Chem. 2018, 45,6343
73. Murie, V. E.; Nishimura, R. H. V.; Rolim, L. A.; Vessecchi, R.; Lopes, N. P.; Clososki, G. C.; J. Org. Chem. 2018, 83, 871 .

74. Comins, D. L.; Nolan, J. M.; Bori, I. D.; Tetrahedron Lett. 2005, 46, 6697.

75. Dumouchel, S.; Mongin, F.; Trécourt, F.; Quéguiner, G.; Tetrahedron Lett. 2003, 44, 2033.

76. Barl, N. M.; Werner, V.; Sämann, C.; Knochel, P.; Heterocycles 2014, $88,827$.

77. Boudet, N.; Lachs, J. R.; Knochel, P.; Org. Lett. 2007, 9, 5525.

78. Nishimura, R. H. V.; Murie, V. E.; Soldi, R. A.; Clososki, G. C.; Synthesis 2015, 47, 1455.

79. Nishimura, R. H. V.; Murie, V. E.; Soldi, R. A.; Lopes, J. L. C.; Clososki, G. C.; J. Braz. Chem. Soc. 2015, 26, 2175.

80. Nishimura, R. H. V.; Vaz, A. L. L.; Bozzini, L. A.; Murie, V. E.; Clososki, G. C.; Tetrahedron 2019, 75, 464.

81. Bao, R. L-Y; Zhao, R.; Shi, L.; Chem. Commun. 2015, 51, 6884

82. Baron, O.; Knochel, P.; Angew. Chem., Int. Ed. 2005, 44, 3133.

83. Zhou, G.; Liu, X.; Liu, X.; Nie, H.; Zhang, S.; Chen, W.; Adv. Synth. Catal. 2013, 355, 3575 .

84. Roering, A. J.; Hale, L. V. A.; Squier, P. A.; Ringgold, M. A.; Wiederspan, E. R.; Clark, T. B.; Org. Lett. 2012, 14, 3558.

85. Roosen, P. C.; Kallepalli, V. A.; Chattopadhyay, B.; Singleton, D. A.; Maleczka, R. E.; Smith, M. R.; J. Am. Chem. Soc. 2012, 134, 11350.

86. Dai, H.-X.; Yu, J.-Q.; J. Am. Chem. Soc. 2012, 134, 134.

87. Larsen, M. A.; Hartwig, J. F.; J. Am. Chem. Soc. 2014, 136, 4287.

88. Tajuddin, H.; Harrisson, P.; Bitterlich, B.; Collings, J. C.; Sim, N.; Batsanov, A. S.; Cheung, M. S.; Kawamorita, S.; Maxwell, A. C.; Shukla, L.; Morris, J.; Lin, Z.; Marder, T. B.; Steel, P. G.; Chem. Sci. 2012, 3, 3505.

89. Wang, Z.; Dong, J.; Hao, Y.; Li, Y.; Liu, Y.; Song, H.; Wang, Q.; J. Org. Chem. 2019, 84, 16245.

90. Dong, J.; Wang, Z.; Wang, X.; Song, H.; Liu, Y.; Wang, Q.; J. Org. Chem. 2019, 84, 7532 .

91. Nagase, M.; Kuninobu, Y.; Kanai, M.; J. Am. Chem. Soc. 2016, 138, 6103.

92. Sahoo, H.; Ramakrishna, I.; Baidya, M.; ChemistrySelect 2016, 1, 1949.

93. Singh, R.; Sran, A.; Carroll, D. C.; Huang, J.; Tsvetkov, L.; Zhou, X.; Sheung, J.; McLaughlin, J.; Issakani, S. D.; Payan, D. G.; Shaw, S. J.; Bioorg. Med. Chem. Lett. 2015, 25, 5199.

94. Motati, D. R.; Uredi, D.; Watkins, E. B.; Chem. Sci. 2018, 9, 1782.

95. Trump, E. L.; Zhou, X.; Trans. Kans. Acad. Sci. 1993, 96, 167.

96. You, Z.; Ran, X.; Dai, Y.; Ran, Y.; J. Mycol. Med. 2018, 28, 492.

97. Proctor, R. S. J.; Phipps, R. J.; Angew. Chem., Int. Ed. 2019, 58, 13666.

98. El Rassi, F.; Khoury, H. J.; Pharmgenomics Pers. Med. 2013, 6, 57.

99. Gnaim, S.; Scomparin, A.; Li, X.; Baran, P. S.; Rader, C.; SatchiFainaro, R.; Shabat, D.; Bioconjug. Chem. 2016, 27, 1965.

100. DiRocco, D. A.; Dykstra, K.; Krska, S.; Vachal, P.; Conway, D. V.; Tudge, M.; Angew. Chem., Int. Ed. 2014, 53, 4802.

101. Moir, M.; Danon, J. J.; Reekie, T. A.; Kassiou, M.; Expert Opin. Drug Discov. 2019, 14, 1137.

102. Venditto, V. J.; Simanek, E. E.; Mol. Pharmaceutics 2010, 7, 307.

103. Zhang, Z.; Tanaka, K.; Yu, J.-Q.; Nature 2017, 543, 538.

104. Jana, R.; Pathak, T. P.; Sigman, M. S.; Chem. Rev. 2011, 111, 1417.

105. Senra, J. D.; Aguiar, L. C. S.; Simas, A. B. C.; Curr. Org. Synth. 2011, $8,53$.

106. Nakao, Y.; Hiyama, T.; Chem. Soc. Rev. 2011, 40, 4893.

107. Maluenda, I.; Navarro, O.; Molecules. 2015, 20, 7528

108. Cordovilla, C.; Bartolomé, C.; Martínez-Ilarduya, J. M.; Espinet, P.; ACS Catal. 2015, 5, 3040.

109. Jagtap, S.; Catalysts 2017, 7, 267.

110. Henry, M. C.; Mostafa, M. A. B.; Sutherland, A.; Synthesis 2017, 49, 4586.

111. Victor, M. M.; Da Silva, G. S. B.; Quim. Nova 2016, 39, 859. 
112. Biajoli, A. F. P.; Schwalm, C. S.; Limberger, J.; Claudino, T. S.; Monteiro, A. L.; J. Braz. Chem. Soc. 2014, 25, 2186.

113. Quasdorf, K. W.; Antoft-Finch, A.; Liu, P.; Silberstein, A. L.; Komaromi, A.; Blackburn, T.; Ramgren, S. D.; Houk, K. N.; Snieckus, V.; Garg, N. K.; J. Am. Chem. Soc. 2011, 133, 6352.

114. Iglesias, M. J.; Prieto, A.; Nicasio, M. C.; Org. Lett. 2012, 14, 4318.

115. Malhotra, S.; Seng, P. S.; Koenig, S. G.; Deese, A. J.; Ford, K. A.; Org. Lett. 2013, 15, 3698.

116. Handa, S.; Wang, Y.; Gallou, F.; Lipshutz, B. H.; Science 2015, 349, 1087.

117. Thapa, S.; Gurung, S. K.; Dickie, D. A.; Giri, R.; Angew. Chem., Int. Ed. 2014, 53, 11620 .

118. Shrestha, B.; Thapa, S.; Gurung, S. K.; Pike, R. A. S.; Giri, R.; J. Org. Chem. 2016, 81, 787.

119. Bellan, A. B.; Kuzmina, O. M.; Vetsova, V. A.; Knochel, P.; Synthesis 2017, 49, 188.

120. Steib, A. K.; Kuzmina, O. M.; Fernandez, S.; Malhotra, S.; Knochel, P.; Chem. - Eur. J. 2015, 21, 1961.

121. Akram, M. O.; Shinde, P. S.; Chintawar, C. C.; Patil, N. T.; Org. Biomol. Chem. 2018, 16, 2865.

122. Baenziger, M.; Pachinger, W.; Stauffer, F.; Zaugg, W.; Org. Process Res. Dev. 2019, 23, 1908.

123. Garrett, C. E.; Prasad, K.; Adv. Synth. Catal. 2004, 346, 889.

124. Toyota, M.; Komori, C.; Ihara, M.; J. Org. Chem. 2000, 65, 7110.

125. Zhang, Y.; Gao, J.; Li, W.; Lee, H.; Lu, B. Z.; Senanayake, C. H.; J. Org. Chem. 2011, 76, 6394.

126. Colomb, J.; Billard, T.; Tetrahedron Lett. 2013, 54, 1471.

127. Pal, R.; Chatterjee, N.; Roy, M.; Nouh, E. S. A.; Sarkar, S.; Jaisankar, P.; Sarkar, S.; Sen, A. K.; Tetrahedron Lett. 2016, 57, 43.

128. Talele, T. T.; J. Med. Chem. 2016, 59, 8712.

129. Reddy, E. A.; Islam, A.; Mukkanti, K.; Venua, B. K.; Pal, M.; J. Braz. Chem. Soc. 2010, 21, 1681.

130. Brad Nolt, M.; Zhao, Z.; Wolkenberg, S. E.; Tetrahedron Lett. 2008, 49 , 3137.

131. Madrid, P. B.; Sherrill, J.; Liou, A. P.; Weisman, J. L.; DeRisi, J. L.; Guy, R. K.; Bioorg. Med. Chem. Lett. 2005, 15, 1015.

132. Duarte, L. F. B.; Barbosa, E. S.; Oliveira, R. L.; Pinz, M. P.; Godoi, B.; Schumacher, R. F.; Luchese, C.; Wilhelm, E. A.; Alves, D.; Tetrahedron Lett. 2017, 58, 3319.

133. Zhou, Y.; Yan, W.; Cao, D.; Shao, M.; Li, D.; Wang, F.; Yang, Z.; Chen, Y.; He, L.; Wang, T.; Shen, M.; Chen, L.; Eur. J. Med. Chem. 2017, 138, 1114.

134. Comins, D. L.; Joseph, S. P. In Comprehensive Heterocyclic Chemistry II; Katritzky, A. R.; Rees, C. W., eds.; Pergamon: Elsevier Science Ltd., 1996, cap. 6.02.

135. Mirjafary, Z.; Saidian, H.; Sahandi, M.; Shojaei, L.; J. Braz. Chem. Soc. 2014, 25, 1253.

136. Fletcher, D. I.; Ganellin, C. R.; Piergentili, A.; Dunn, P. M.; Jenkinson, D. H.; Bioorg. Med. Chem. 2007, 15, 5457.
137. Pérez, B. C.; Fernandes, I.; Mateus, N.; Teixeira, C.; Gomes, P.; Bioorg. Med. Chem. Lett. 2013, 23, 6769.

138. Sánchez-Martín, R.; Campos, J. M.; Conejo-García, A.; Cruz-López, O.; Báñez-Coronel, M.; Rodríguez-González, A.; Gallo, M. A.; Lacal, J. C.; Espinosa, A.; J. Med. Chem. 2005, 48, 3354.

139. Barbosa-Lima, G.; da Silveira Pinto, L. S.; Kaiser, C. R.; Wardell, J. L.; De Freitas, C. S.; Vieira, Y. R.; Marttorelli, A.; Cerbino Neto, J.; Bozza, P. T.; Wardell, S. M. S. V.; de Souza, M. V. N.; Souza, T. M. L.; Eur. J. Med. Chem. 2017, 127, 434.

140. Sinha, M.; Dola, V. R.; Soni, A.; Agarwal, P.; Srivastava, K.; Haq, W.; Puri, S. K.; Katti, S. B.; Bioorg. Med. Chem. 2014, 22, 5950.

141. Serafinowska, H. T.; Blaney, F. E.; Lovell, P. J.; Merlo, G. G.; Scott, C. M.; Smith, P. W.; Starr, K. R.; Watson, J. M.; Bioorg. Med. Chem. Lett. 2008, 18, 5581.

142. Rogers, D. A.; Bensalah, A. T.; Espinosa, A. T.; Hoerr, J. L.; Refai, F. H.; Pitzel, A. K.; Alvarado, J. J.; Lamar, A. A.; Org. Lett. 2019, 21, 4229.

143. Chambers, R. D.; Holling, D.; Sandford, G.; Batsanov, A. S.; Howard, J. A. K.; J. Fluor. Chem. 2004, 125, 661.

144. Paloque, L.; Verhaeghe, P.; Casanova, M.; Castera-Ducros, C.; Dumètre, A.; Mbatchi, L.; Hutter, S.; Kraiem-M'Rabet, M.; Laget, M.; Remusat, V.; Rault, S.; Rathelot, P.; Azas, N.; Vanelle, P.; Eur. J. Med. Chem. 2012, 54, 75.

145. Kitamura, M.; Fukuma, H.; Kobayashi, M.; Okayama, S.; Okauchi, T.; J. Org. Chem. 2016, 81, 3956.

146. Colomb, J.; Becker, G.; Fieux, S.; Zimmer, L.; Billard, T.; J. Med. Chem. 2014, 57, 3884.

147. Machado, A. H. L.; Pandoli, O.; De Mariz e Miranda, L. S.; De Souza, R. O. M. A.; Rev. Virtual Quim. 2014, 6, 1076.

148. Baxendale, I. R.; J. Chem. Technol. Biotechnol. 2013, 88, 519.

149. Baumann, M.; Baxendale, I. R.; Beilstein J. Org. Chem. 2015, 11, 1194.

150. Pinho, V. D.; Miranda, L. S. M.; De Souza, R. O. M. A.; Rev. Virtual Quim. 2015, 7, 144.

151. De Souza, R. O. M. A.; Miranda, L. S. M.; Rev. Virtual Quim. 2014, 6, 34.

152. Baumann, M.; Baxendale, I. R.; J. Org. Chem. 2015, 80, 10806.

153. Cravotto, G.; Bonrath, W.; Tagliapietra, S.; Speranza, C.; Gaudino, E. C.; Barge, A.; Chemical Engineering and Processing: Process Intensification 2010, 49, 930.

154. Yalgin, H.; Luart, D.; Len, C.; J. Flow Chem. 2016, 6, 80.

155. Lengyel, L.; Nagy, T. Z.; Sipos, G.; Jones, R.; Dormán, G.; Ürge, L.; Darvas, F.; Tetrahedron Lett. 2012, 53, 738.

156. Di Filippo, M.; Baumann, M.; Eur. J. Org. Chem. 2020, 2020, 6199.

157. Therkelsen, M.; Rasmussen, M. T.; Lindhardt, A. T.; Chem. Commun. 2015, 51, 9651.

158. Chopra, R.; Chibale, K.; Singh, K.; Eur. J. Med. Chem. 2018, 148, 39.

159. Qi, H.; Li, X.; Liu, Z.; Miao, S. S.; Fang, Z.; Chen, L.; Fang, Z.; Guo, K.; ChemistrySelect 2018, 3, 10689. 